\title{
A Window of Vascular Plasticity Coupled to Behavioral Recovery after Stroke
}

\author{
${ }^{\circledR}$ Michael R. Williamson, ${ }^{1}$ Ronald L. Franzen, ${ }^{2}$ Cathleen Joy A. Fuertes, ${ }^{2}$ Andrew K. Dunn, ${ }^{1,3}$ \\ Michael R. Drew, ${ }^{1,4}$ and ${ }^{-}$Theresa A. Jones ${ }^{1,2}$ \\ ${ }^{1}$ Institute for Neuroscience, ${ }^{2}$ Department of Psychology, ${ }^{3}$ Department of Biomedical Engineering, and ${ }^{4}$ Center for Learning and Memory and \\ Department of Neuroscience, University of Texas at Austin, Austin, Texas 78712
}

Stroke causes remodeling of vasculature surrounding the infarct, but whether and how vascular remodeling contributes to recovery are unclear. We established an approach to monitor and compare changes in vascular structure and blood flow with high spatiotemporal precision after photothrombotic infarcts in motor cortex using longitudinal 2-photon and multiexposure speckle imaging in mice of both sexes. A spatially graded pattern of vascular structural remodeling in peri-infarct cortex unfolded over the first 2 weeks after stroke, characterized by vessel loss and formation, and selective stabilization of a subset of new vessels. This vascular structural plasticity was coincident with transient activation of transcriptional programs relevant for vascular remodeling, reestablishment of peri-infarct blood flow, and large improvements in motor performance. Local vascular plasticity was strongly predictive of restoration of blood flow, which was in turn predictive of behavioral recovery. These findings reveal the spatiotemporal evolution of vascular remodeling after stroke and demonstrate that a window of heightened vascular plasticity is coupled to the reestablishment of blood flow and behavioral recovery. Our findings support that neovascularization contributes to behavioral recovery after stroke by restoring blood flow to peri-infarct regions. These findings may inform strategies for enhancing recovery from stroke and other types of brain injury.

Key words: angiogenesis; blood flow; in vivo imaging; neural repair; recovery; vascular remodeling

Significance Statement

An improved understanding of neural repair could inform strategies for enhancing recovery from stroke and other types of brain injury. Stroke causes remodeling of vasculature surrounding the lesion, but whether and how the process of vascular remodeling contributes to recovery of behavioral function have been unclear. Here we used longitudinal in vivo imaging to track vascular structure and blood flow in residual peri-infarct cortex after ischemic stroke in mice. We found that stroke created a restricted period of heightened vascular plasticity that was associated with restoration of blood flow, which was in turn predictive of recovery of motor function. Therefore, our findings support that vascular remodeling facilitates behavioral recovery after stroke by restoring blood flow to peri-infarct cortex.

\section{Introduction}

The cerebral vasculature distributes nutrients to support metabolically demanding neural activity. Ischemic stroke interrupts the vascular supply to part of the brain and produces an infarct surrounded by a surviving but metabolically and functionally impaired peri-infarct region (Carmichael et al., 2004; Summers

Received June 9, 2020; revised Aug. 3, 2020; accepted Aug. 25, 2020.

Author contributions: M.R.W. designed research; M.R.W., R.L.F., and C.J.A.F. performed research; M.R.W.,

R.L.F., C.J.A.F., A.K.D., M.R.D., and T.A.J. analyzed data; M.R.W. wrote the first draft of the paper; M.R.W.,

R.L.F., C.J.A.F., A.K.D., M.R.D., and T.A.J. edited the paper; M.R.W. wrote the paper.

The authors declare no competing financial interests.

This work was supported by Canadian Institutes of Health Research Doctoral Award DFS-157838 to M.R.W., and National Institutes of Health R01 NS108484 and R01 EB011556 to A.K.D., R01 MH102595 and R01 MH117426 to M.R.D., and R37 NS056839 to T.A.J.

Correspondence should be addressed to Michael R. Williamson at mrwillia@utexas.edu.

https://doi.org/10.1523/JNEUROSCI.1464-20.2020

Copyright $\odot 2020$ the authors et al., 2017). Structural and functional reorganization of multiple cell types in residual peri-infarct tissue is critical for poststroke behavioral recovery (Carmichael, 2006; Jones and Adkins, 2015; Tennant et al., 2017; Kim et al., 2018). However, effective neural repair and subsequent behavioral recovery depend on the reestablishment of a microenvironment capable of supporting such reorganization.

Stroke-induced vascular remodeling is hypothesized to support the recovery of more normal neuronal function and neural repair by restoring blood flow, thereby resolving metabolic stress and enabling structural and functional changes in other cells (Rust et al., 2019b). Across several animal models, peri-infarct blood flow deficits are most pronounced soon after ischemia (Schrandt et al., 2015; Clark et al., 2019b). While blood flow deficits, at least over relatively large volumes, tend to resolve by $\sim 1$ week after stroke (Lin et al., 2002; Schrandt et al., 2015; Clark et al., 2019b), localized abnormalities in neurovascular 
coupling and neuronal function persist for at least several weeks (Carmichael et al., 2004; Brown et al., 2009; Lake et al., 2017; Summers et al., 2017; He et al., 2020). The formation of new vessels could help to restore blood flow to ischemic regions and alleviate regional perfusion deficits. Histologic evidence for vascular structural plasticity has been found in stroke models (Lin et al., 2002; Dijkhuizen et al., 2003; Ergul et al., 2012; Martín et al., 2012; Liu et al., 2014; Lake et al., 2017; Clark et al., 2019b; Rust et al., 2019a,c) and human stroke (Krupinski et al., 1994; Szpak et al., 1999). Increases in vascular density and colabeling of vessels with proliferation markers within peri-infarct cortex have been found within the first several weeks after stroke (Dijkhuizen et al., 2003; Martín et al., 2012; Liu et al., 2014; Lake et al., 2017; Clark et al., 2019b; Rust et al., 2019a). However, the assumption that changes in vascular structure underlie blood flow restoration and subsequent behavioral recovery is largely based on the rough temporal coincidence of histologic evidence of structural vascular changes, reestablishment of peri-infarct blood flow, and behavioral improvement (Lin et al., 2002; Dijkhuizen et al., 2003; Ergul et al., 2012; Liman and Endres, 2012; Yanev and Dijkhuizen, 2012; Lake et al., 2017; Clark et al., 2019b; Rust et al., 2019b).

Whether and how vascular remodeling contributes to recovery of behavioral function have been unclear. Our goal was to define the poststroke progression and association of vascular structural plasticity, blood flow, and behavioral recovery. We applied longitudinal multimodal in vivo imaging to track the evolution of vascular structural plasticity and blood flow in a mouse model of focal ischemic stroke. 2-Photon (2P) imaging of endothelial cells allowed us to visualize structural changes at single capillary resolution across time and space. Concurrent multiexposure speckle imaging (MESI) produced blood flow maps that we could relate to structural plasticity within defined regions. We found that vascular structure was remarkably stable over months in naive mice. In contrast, stroke instigated a period of substantial change in the structure of the vascular network within peri-infarct cortex, including formation and elimination of capillary segments. This structural plasticity was spatially graded and restricted to the first 2 weeks after stroke, coincident with reestablishment of peri-infarct blood flow. Crucially, the magnitude of structural vascular plasticity was strongly correlated with the reestablishment of blood flow. Subsequently, we found that the degree to which peri-infarct blood flow was restored predicted forelimb motor function on a day-to-day basis. These findings support the hypothesis that vascular structural plasticity facilitates behavioral recovery after stroke by restoring blood flow to peri-infarct regions.

\section{Materials and Methods}

Subjects. Three- to 6-month-old (18-29 g) Tie2-GFP mice (GFP-labeled endothelial cells, JAX stock \#003658) on an FVB background and WT C57BL/6J mice of approximately equal numbers of each sex were used. Mice were bred locally. Animals were housed 2-5 per cage in a room with lights on at 07:00 to 19:00. Animals had free access to food and water, except during training and testing on the single-seed reaching task when they were placed on restricted feeding to maintain $\sim 90 \%$ free feeding weight. Protocols were approved by an Institutional Animal Care and Use Committee. Sample sizes were based on previous published (Schrandt et al., 2015; Clark et al., 2019b) and unpublished work using MESI and the single-seed task, as well as calculations from pilot data to determine the minimum number of animals needed to achieve a power level of $80 \%$ for key measures.

Experimental design. We performed three experiments. First, we used 2P imaging and MESI in Tie2-GFP mice $(N=6)$ to assess the stability of cortical vascular structure and cerebral blood flow in the intact brain over a period of 3 months. Second, we used 2P imaging and MESI to track vascular structure and blood flow before and after photothrombotic cortical infarcts in Tie2-GFP mice $(N=6)$. Tie2-GFP mice are useful for imaging vascular structure but become blind early in life. A cohort of Tie2-GFP mice failed to establish proficiency on our skilled reaching task. Rodents normally do not depend on vision to perform skilled reaching tasks but could conceivably do so in the presence of forelimb sensorimotor impairments induced by infarcts (HermerVazquez et al., 2007). Therefore, for the third experiment, we used C57BL/6J mice. We repeatedly imaged blood flow with MESI and assayed motor function with the single-seed reaching task to relate the reestablishment of blood flow with motor recovery $(N=12)$. In the last experiment, 1 mouse died after photothrombosis, 1 was euthanized because of skull regrowth under the window, and 1 was excluded from behavioral measures because of a lack of behavioral impairment after photothrombosis (success rate $>100 \%$ of baseline performance on day 3).

Cranial windows. Cranial windows were created over the forelimb region of motor cortex as before (Holtmaat et al., 2009; Clark et al., 2019b). Mice were anesthetized with isoflurane (3\% induction, $1 \%-2 \%$ maintenance) in oxygen and placed in a stereotaxic frame. Dexamethasone $(2 \mathrm{mg} /$ $\mathrm{kg})$ and carprofen $(2.5 \mathrm{mg} / \mathrm{kg})$ were given subcutaneously. A craniotomy $(\sim 4.5 \mathrm{~mm})$ centered $1.5 \mathrm{~mm}$ lateral to bregma was made with a dental drill leaving the dura intact. Saline-soaked Gelfoam (Pfizer) was used to control bleeding. Cyanoacrylate and dental cement were used to secure a 4-mm-diameter \#1 cover glass (Warner Instruments) in place. Buprenorphine $(0.05 \mathrm{mg} / \mathrm{kg}$, s.c.) was given for pain management. Carprofen $(2.5 \mathrm{mg} / \mathrm{kg})$ was given daily for 1 week to limit inflammation.

$2 \mathrm{P}$ imaging and analysis. $2 \mathrm{P}$ imaging was used to image cortical vasculature. Mice were anesthetized with isoflurane (3\% induction, $\sim 1.5 \%$ maintenance) in oxygen and placed in a frame. During some sessions, $40 \mu \mathrm{l}$ of $1 \% \mathrm{w} / \mathrm{v} 70 \mathrm{kDa}$ Texas Red-conjugated dextran (Invitrogen, D1830) was injected retro-orbitally to label plasma. Body temperature was maintained with a heated pad. $2 \mathrm{P}$ imaging was performed using a mode-locked femtosecond multiphoton microscope (MaiTai, Spectra Physics) tuned to $870 \mathrm{~nm}$. Image stacks were acquired with $512 \times 512$ pixel resolution and $2.0 \mu \mathrm{m} z$ step size using a water-immersion $20 \times 1$ $1.0 \mathrm{NA}$ objective (Olympus). A Pockels cell adjusted laser power with imaging depth to maintain fluorescence intensity along the $z$ axis (Clark et al., 2019a). Fluorescent emission was detected by photomultiplier tubes after passing through a $595 \pm 35 \mathrm{~nm}$ (Texas Red) or $525 \pm 25 \mathrm{~nm}$ (GFP) bandpass filter. The pattern of leptomeningeal vessels from widefield speckle images was used to identify the same regions across imaging days.

Vessel segments were defined as the region between vascular branch points. In order to quantify changes in vascular structure, we applied methodology commonly used to assess dendritic spine plasticity (Xu et al., 2009; Clark et al., 2019a). $z$-stack images collected on separate days were analyzed simultaneously. Segments were counted as stable if they appeared in both the currently analyzed image as well as that from the previous time point. Segments were classified as new if not present in the image from the previous time point. Eliminated segments were counted if not present in the current image but were present in that of the previous time point. The percentage of new or eliminated segments at a given time point was calculated relative to the total number of segments from the previous time point. A newly formed segment was defined as persistent if it was visible at subsequent time points. The proportion of persistent segments is presented relative to the total number of new segments formed at a given time point. We did not analyze regions within the infarct core, which was determined by MESI at day 2 (parenchymal flow $<20 \%$ baseline flow) (Clark et al., 2019a,b) and confirmed by bright autofluorescence in $2 \mathrm{P}$ images. Distance from the infarct border was measured from the center of the image. Vessel segments of each type (capillary, venule, arteriole) were analyzed separately. Capillaries were defined by cross-sectional diameter 3-9.5 $\mu \mathrm{m}$ measured using GFP fluorescence. Penetrating arterioles and venules, regardless of diameter, were not counted as capillaries. Regions with poor visibility (e.g., beneath a large superficial pial vessel) were excluded from analysis. Images are presented as maximum or average intensity projections, except where otherwise stated. 
A
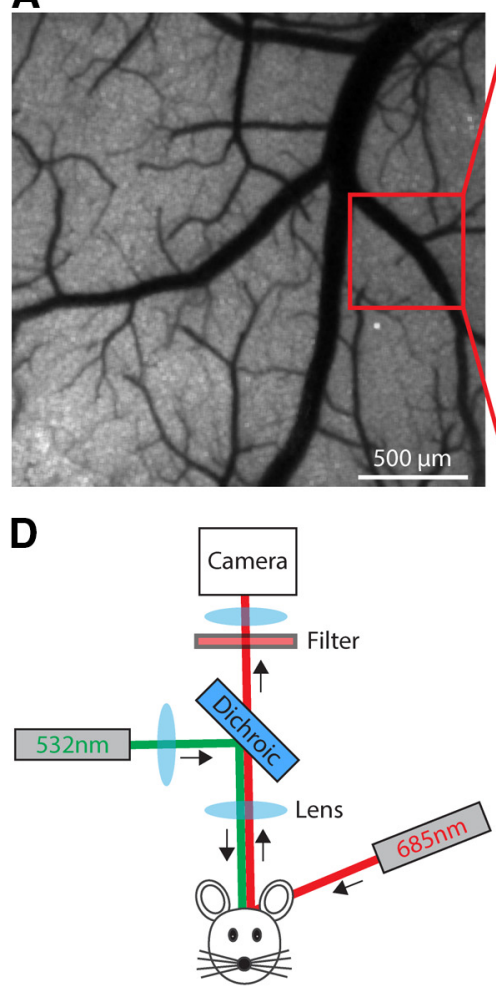

B

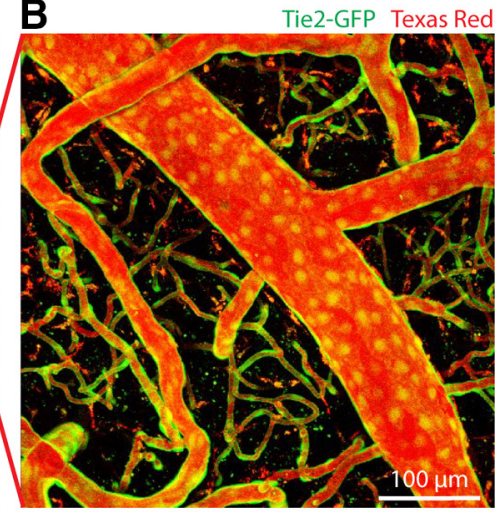

E

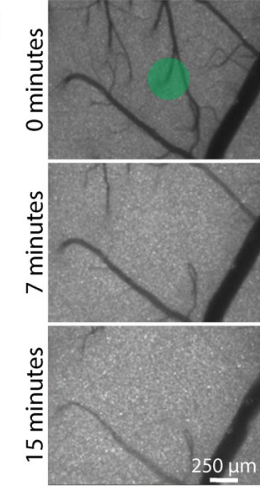

$\mathbf{F}$

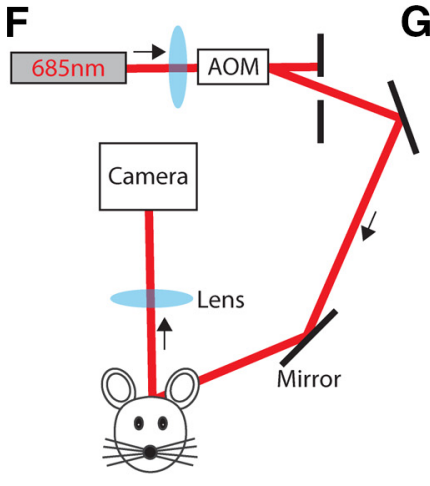

G
C
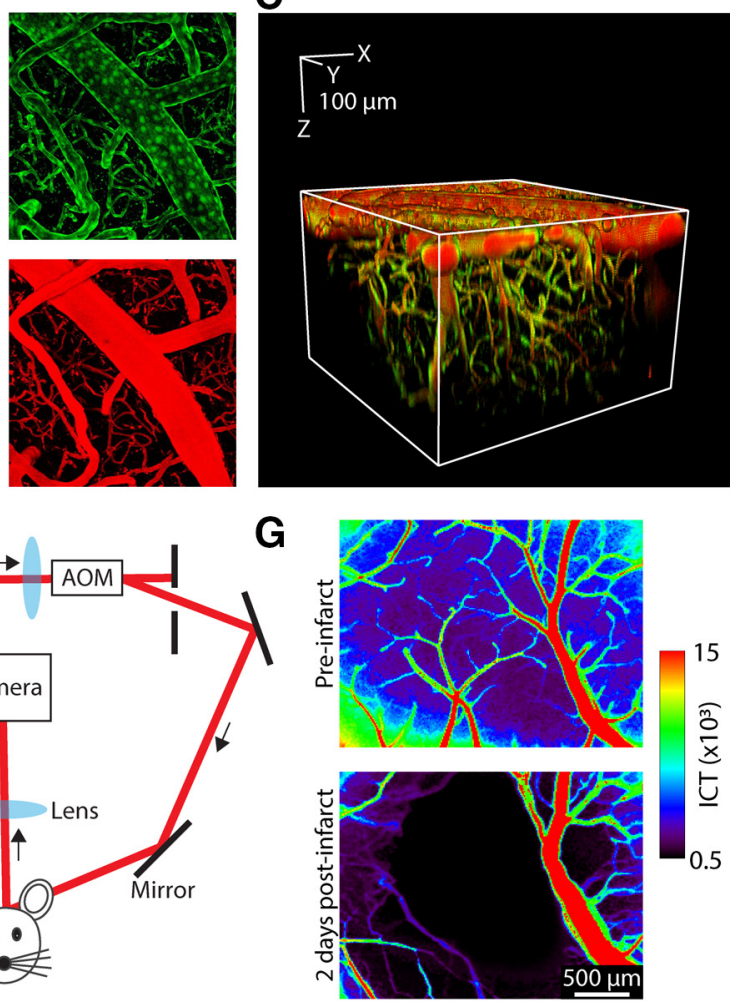

Figure 1. Optical methods. $\boldsymbol{A}, \mathrm{A}$ laser speckle contrast image of the surface of motor cortex through a cranial window. Darker pixels represent higher blood flow. $\boldsymbol{B}$, In vivo $2 \mathrm{P}$ image of the superficial cerebral vasculature from the region indicated in $\boldsymbol{A}$ (50 $\mu \mathrm{m}$ depth). Vessels are labeled with genetically encoded GFP (Tie2-GFP) and intravenous Texas Red-conjugated dextran. $\boldsymbol{C}$, $3 \mathrm{D}$ projection of cortical vasculature from the region indicated in $\boldsymbol{A}$ to a depth of $300 \mu \mathrm{m}$. $\boldsymbol{D}$, Schematic diagram of the instrument used for inducing photothrombotic infarcts with live laser speckle contrast imaging. $\boldsymbol{E}$, Example laser speckle contrast images before, during, and after photothrombosis. Green circle represents the photothrombotic (green laser) target, which is centered on a penetrating arteriole. $\boldsymbol{F}$, Schematic diagram of the instrument used for MESI. AOM, Acousto-optic modulator. $\boldsymbol{G}$, Example of MESI before (top) and $2 \mathrm{~d}$ after (bottom) photothrombosis.

For analysis of stalled capillaries, two-channel Tie2-GFP and Texas Red $z$ stacks with $2 \mu \mathrm{m}$ optical sections were manually searched for capillary segments (GFP channel) lacking blood cell movement (visible in the Texas Red channel as an absence of streaking blood cells, which exclude the Texas Red label) (Villringer et al., 1994; Reeson et al., 2018; Cruz Hernández et al., 2019). Each segment was visible for at least $5.4 \mathrm{~s}$. We classified a capillary as stalled if there was no flow during the entire time it was visible. We present the proportion of stalled capillaries relative to the total number of counted capillary segments per animal.

MESI. MESI was used to collect quantitative images of cortical blood flow (Kazmi et al., 2013; Schrandt et al., 2015; Clark et al., 2019b). Imaging sessions lasted $<10 \mathrm{~min}$. Mice were anesthetized with isoflurane ( $3 \%$ induction, $1.25 \%$ maintenance) in oxygen and headfixed. The same oxygen flow rate and amount of isoflurane were used for each imaging session to minimize effects of anesthesia on blood flow. Body temperature was maintained with a heated pad. The craniotomy was illuminated obliquely with a $685 \mathrm{~nm}$ laser (see Fig. $1 F$ ). Backscattered light was collected by a camera (Basler) over 15 exposure durations ranging from 0.05 to $80 \mathrm{~ms}$. An acousto-optic modulator controlled the amplitude of illumination. Raw images were processed with MATLAB to produce inverse correlation time (ICT) images (Schrandt et al., 2015), where ICT is proportional to blood flow (Clark et al., 2019b). Two baseline images were collected on different days at least 3 weeks after cranial windows were implanted, but before photothrombosis. Additional images were collected on multiple days after photothrombosis dependent on the experiment, typically days $2,5,7,14,21$, and 28 .

Processed ICT images were quantified using ImageJ. For baseline images, the mean ICT values from 8 to 10 discrete, randomly sampled parenchymal regions were averaged to yield a baseline parenchymal flow value. The infarct border was defined from day 2 MESI using a threshold of $20 \%$ of baseline parenchymal flow, which we have found corresponds to histologically defined lesions (Clark et al., 2019b). Peri-infarct blood flow was quantified by averaging the mean ICT values from 8-10 discrete, randomly sampled parenchymal regions within $\sim 500 \mu \mathrm{m}$ of the infarct border and was expressed as a percentage of baseline parenchymal blood flow. The same regions were measured in all postinfarct images and were identified using multiple surface vessels as landmarks. For analysis of parenchymal flow within $2 \mathrm{P}$-imaged regions, flow in each parenchymal region corresponding to a single $2 \mathrm{P}$ image was measured separately. Poststroke blood flow deficits and restoration were not different between Tie2-GFP and C57BL/6J mice (two-way repeated measures ANOVA, strain main effect: $\left.F_{(1,14)}=0.007, p=0.936\right)$.

Focal ischemia. Photothrombotic infarcts were produced in the forelimb area of motor cortex (see Fig. 1D,E) (Clark et al., 2019a,b). Mice were anesthetized with isoflurane (3\% induction, $1.5 \%$ maintenance) in oxygen and placed in a frame. Body temperature was maintained with a heated pad. Pial vessels were visualized with real-time laser speckle contrast imaging. A penetrating arteriole supplying the forelimb motor region (Tennant et al., 2011) was identified, and $0.15 \mathrm{ml}$ of $15 \mathrm{mg} / \mathrm{kg}$ rose Bengal was given intraperitoneally. 3 min later, a $20 \mathrm{~mW} 532 \mathrm{~nm}$ laser was used to illuminate a $\sim 300 \mu \mathrm{m}$ diameter region centered on the chosen penetrating arteriole for $15 \mathrm{~min}$ (Schrandt et al., 2015; Clark et al., 2019b). Successful occlusion was confirmed by speckle imaging.

Behavioral testing. The single-seed reaching task was used to examine upper extremity impairments caused by cortical infarcts. This task is sensitive to deficits in skilled forelimb use and has been described in detail previously (Xu et al., 2009; Clark et al., 2019b). During the shaping phase, animals were placed in a clear Plexiglas chamber with access to a tray of millet seeds through a 4-mm-wide opening. The preferred paw for reaching was defined as the paw used for the majority of reach attempts across $15-30$ reach attempts during a 15 min session. Beginning 2 weeks after cranial window surgery, animals were trained to use their 
A
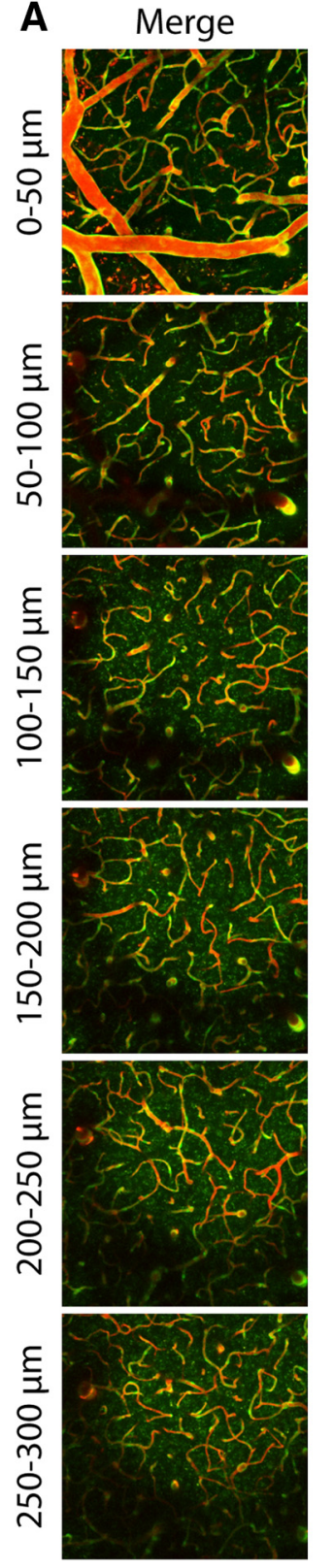

Texas Red
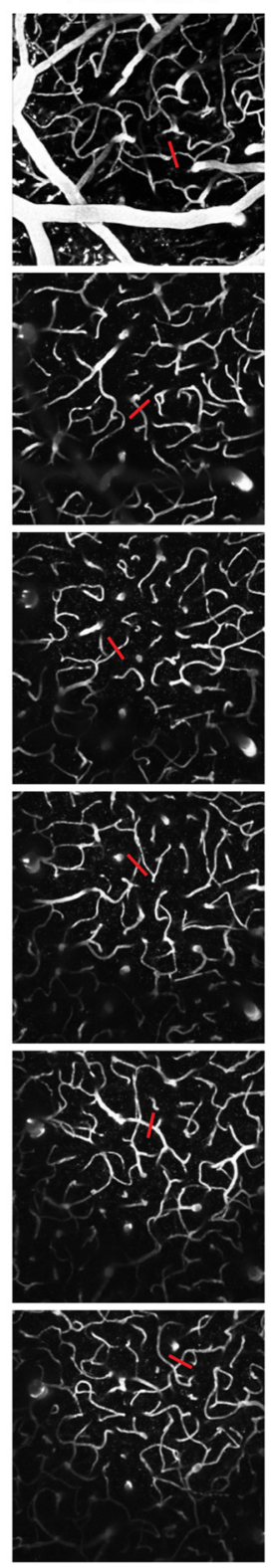

Tie2-GFP
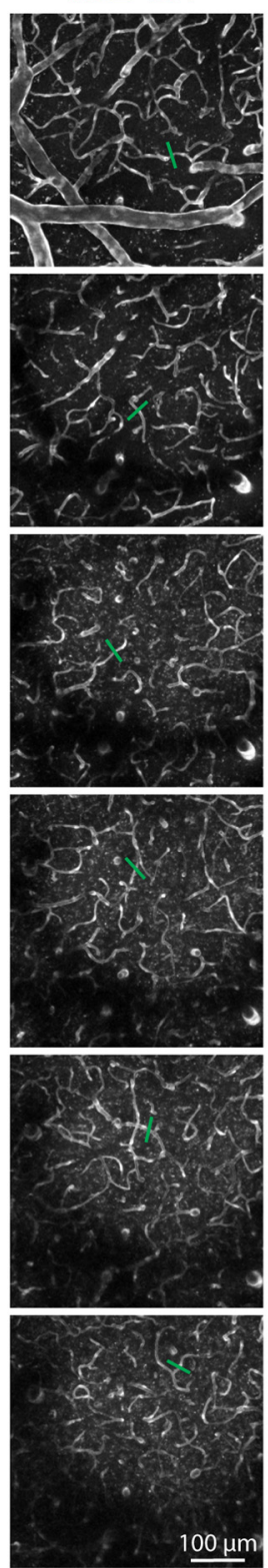

B
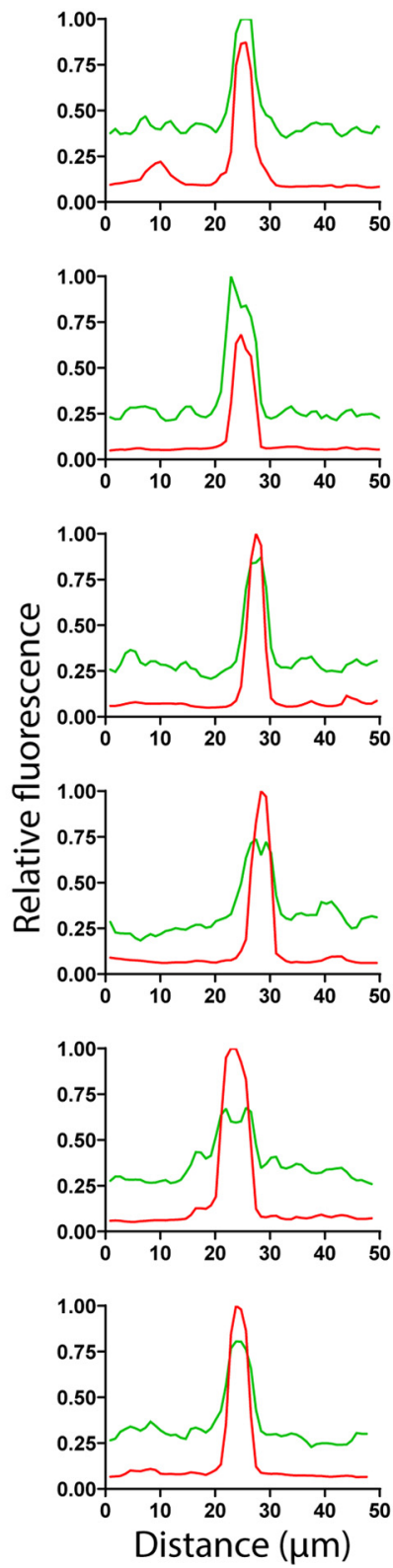

Figure 2. Comparison of in vivo 2P imaging of vasculature with intravascular Texas Red-conjugated dextran and genetically encoded endothelial cell GFP expression (Tie2-GFP). $\boldsymbol{A}$, Maximum intensity projection images $(50 \mu \mathrm{m})$ of cortical vasculature spanning from the pial surface to $300 \mu \mathrm{m}$ deep. $\boldsymbol{B}$, Line profiles of fluorescence intensity across representative capillaries by depth. Measured regions are indicated with red (Texas Red) and green (Tie2-GFP) lines in $\boldsymbol{A}$.

preferred paw (contralateral to the window) to reach for a single millet seed placed on a platform outside the chamber. Training was done during 5 sessions per week for 2 consecutive weeks. Training and testing sessions consisted of up to 30 trials and lasted up to $15 \mathrm{~min}$. Trials were scored as successes or failures. A trial was defined as up to 2 consecutive reach attempts. If the first reach attempt resulted in missing the seed, a second consecutive attempt was allowed. If the second attempt resulted in another miss, knocking the pellet out of its well, or dropping the seed before bringing it to the mouth, the trial was scored a failure. Knocking the pellet out of its well or dropping the seed before bringing it to the mouth on the first attempt was also scored as a failure. If either reach attempt resulted in the mouse grasping the seed and bringing it to its mouth, the trial was scored a success. Test sessions were done on days 3 , $5,7,14,21$, and 28 after photothrombosis.

Tissue processing and histology. Mice were given Euthasol $(0.1 \mathrm{ml}$, i.p.) and transcardially perfused with $0.1 \mathrm{M} \mathrm{PB}$ followed by $4 \%$ PFA in
PB. Brains were postfixed overnight and sectioned coronally with a vibratome (VT1000S, Leica Microsystems) at $35 \mu \mathrm{m}$ thickness.

One set of every fifth section was Nissl-stained. Cortical area of each hemisphere was determined with ImageJ, and Cavalieri's method was used to estimate cortical volume as the summed area of cortex across sections multiplied by distance between section planes. Lesion volume was calculated as the difference in contralesional and ipsilesional cortical volume (Kim et al., 2018; Clark et al., 2019b).

C57BL/6 J mice were retro-orbitally injected with $0.1 \mathrm{ml}$ of DyLight 488-conjugated tomato lectin (Vector Laboratories, DL1174) $5 \mathrm{~min}$ before perfusion to label perfused vasculature $28 \mathrm{~d}$ after infarct (Robertson et al., 2015). At least four free-floating sections per animal spaced $175 \mu \mathrm{m}$ were stained with isolectin B4 as before (Wälchli et al., 2015; Clark et al., 2019b). Sections were washed three times for $5 \mathrm{~min}$ in PBS, incubated in $50 \mathrm{mM} \mathrm{NH}_{4} \mathrm{Cl}$ for $30 \mathrm{~min}, 80^{\circ} \mathrm{C}$ $50 \mathrm{~mm}$ glycine for $5 \mathrm{~min}$, and $0.3 \%$ Triton for $10 \mathrm{~min}$. Sections were 

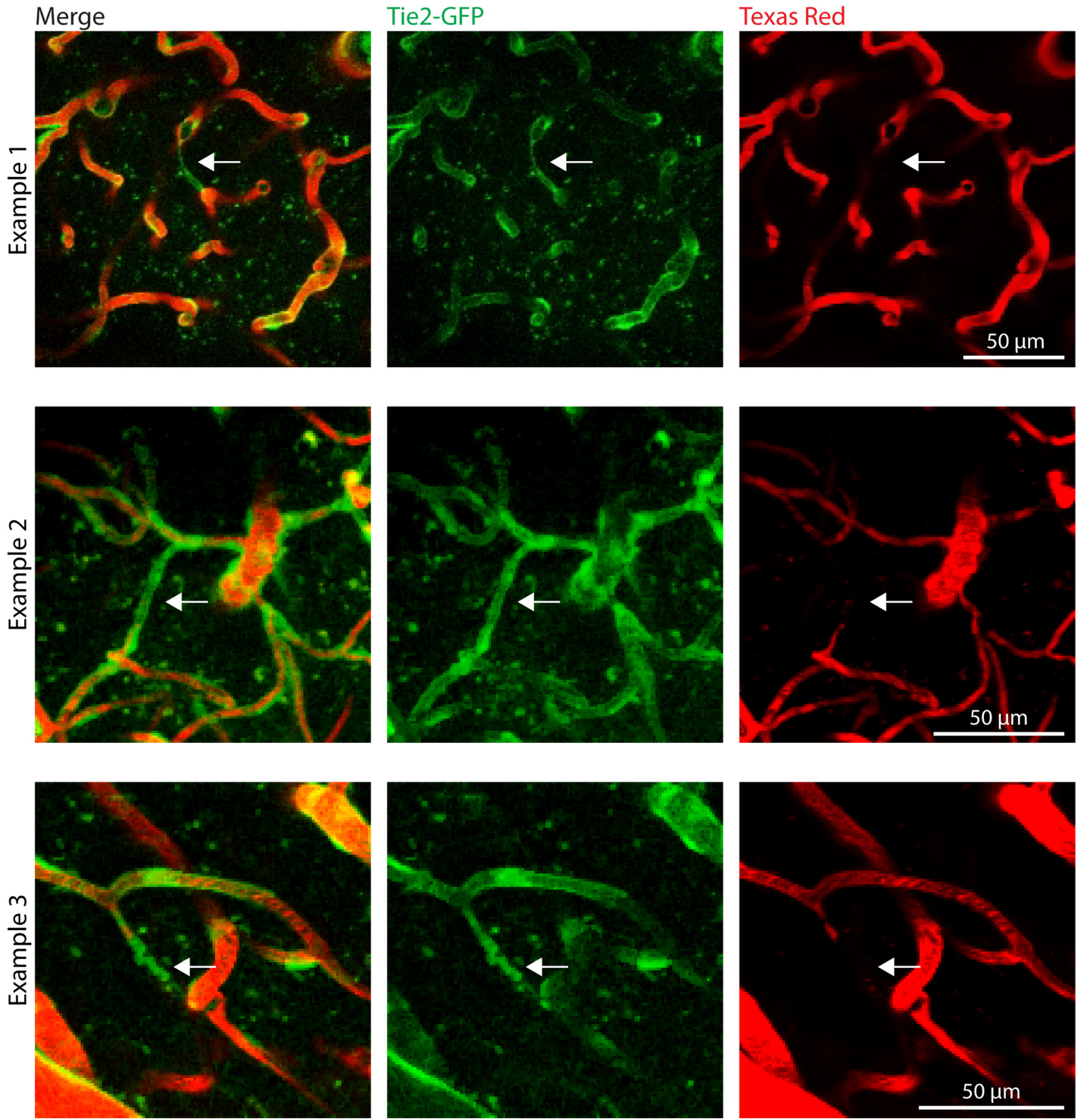

Arrow $=$ vessel segment not labeled by intravascular dye

Figure 3. Incomplete labeling of vasculature with intravascular dye. Three examples of capillary segments visible by GFP expression but not intravascular dye. Some segments completely lacked intravascular dye, whereas others were partially filled. Images are 30-50 $\mu \mathrm{m}$ projections.

then incubated in blocking solution $\left(50 \mathrm{~mm} \mathrm{CaCl}_{2}, 50 \mathrm{~mm} \mathrm{MgCl}, 50\right.$ $\mathrm{mM} \mathrm{MnCl}_{2}, 0.05 \%$ Triton, 2\% goat serum in PBS) with 1:50 biotinylated isolectin B4 (Vector Laboratories, B-1205) for $72 \mathrm{~h}$ at $4^{\circ} \mathrm{C}$. After washing with PBS, sections were incubated in blocking solution with 1:500 AlexaFluor-647-conjugated streptavidin (Jackson ImmunoResearch Laboratories, 016-600-084) overnight at $4^{\circ} \mathrm{C}$, washed, and mounted.

Images were acquired with a TCS SP5 confocal microscope (Leica Microsystems, $20 \times / 0.7$ NA objective); $1024 \times 1024$ pixel images were collected with $2 \mu \mathrm{m} z$-step size and 3-frame averaging. Acquisition parameters were consistent across all samples and images. Images were analyzed using ImageJ. Maximum intensity projections were uniformly processed with a custom macro. A Gaussian filter $(\sigma=1)$ was applied. Then, background was subtracted using a 15 pixel rolling ball radius with the sliding paraboloid option checked. Images were then thresholded and denoised. The area fraction of binarized images was calculated with the Analyze Particles tool. For measurement of branches and vascular length from images of tomato lectin-labeled vasculature, the Skeletonize tool was applied to binary images and the Analyze Skeleton tool was used to analyze the resulting skeletons.

Analysis of an endothelial cell transcriptome resource. We analyzed data from a resource in which the brain endothelial cell transcriptome was sequenced in health and disease (Munji et al., 2019). Gene expression changes were evaluated from peri-infarct endothelial cells isolated 3 


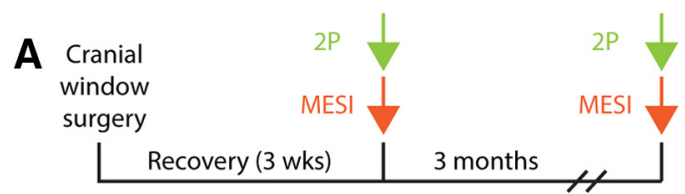

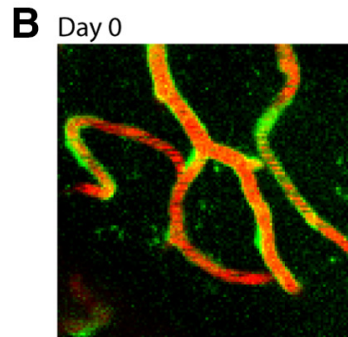

Tie2-GFP Texas Red

D Day 0

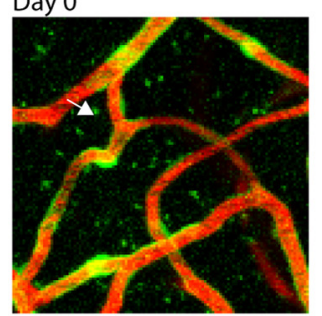

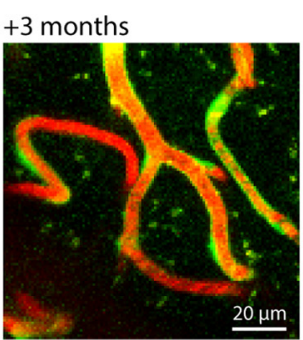

Stable

+3 months

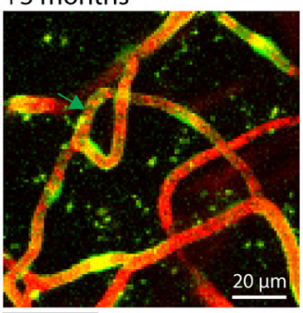

4 New

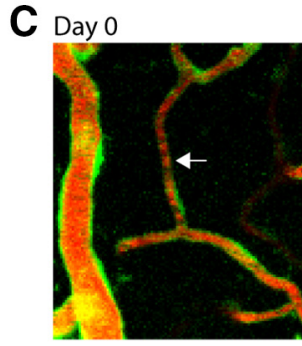

E

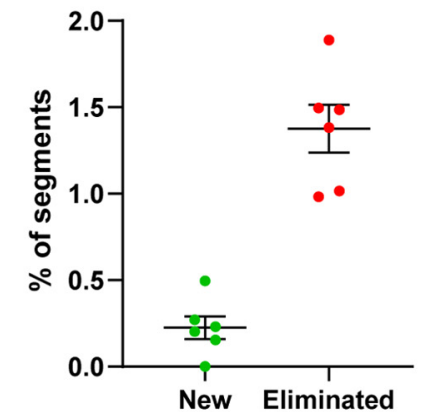

$\mathbf{F}$
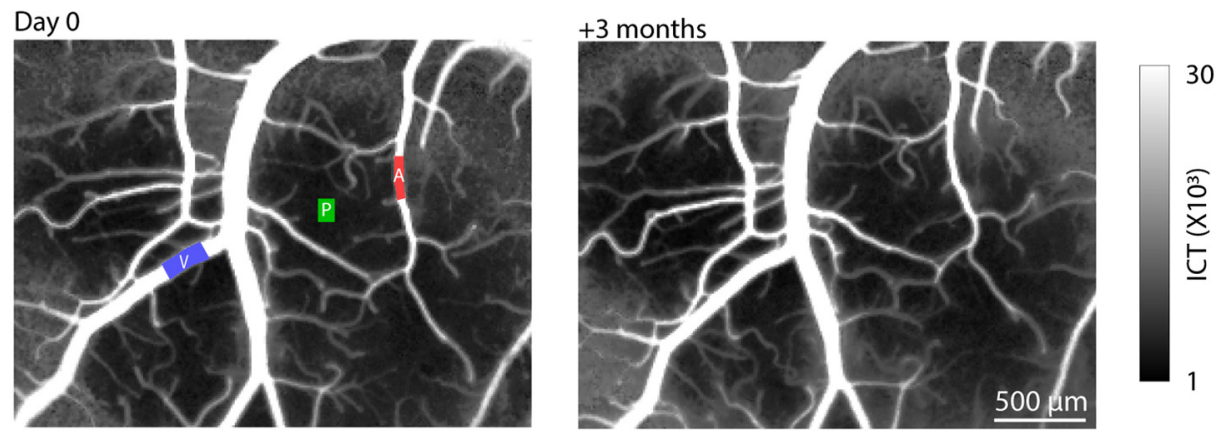

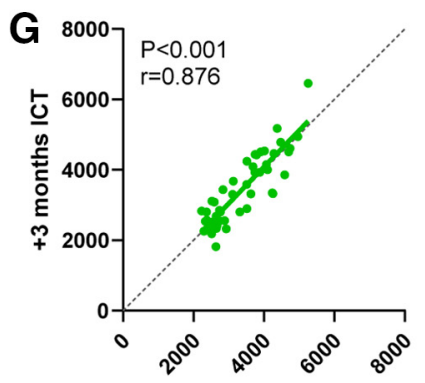

Day 0 ICT

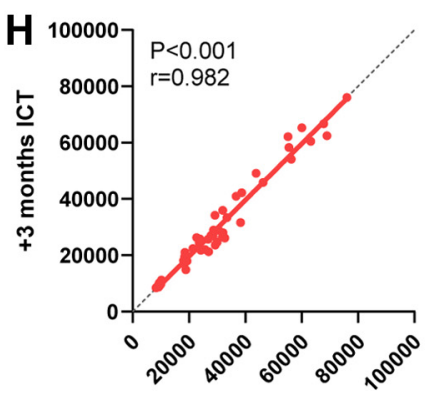

Day 0 ICT

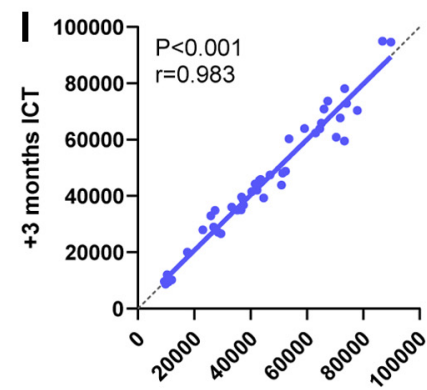

Day 0 ICT

Figure 4. Stability of vascular structure and blood flow in intact cortex. $\boldsymbol{A}$, Timeline for repeated imaging of vascular structure (2P) and blood flow (MESI). $\boldsymbol{B}, 50 \mu \mathrm{m}$ projection images of stable vessels taken 3 months apart. C, Example of an eliminated capillary (arrows). D, Example of a newly formed capillary (arrows). $\boldsymbol{E}$, Quantification of capillary segment changes over the 3 month interval. Percentages are relative to the total number of capillaries at the first imaging time point. $\boldsymbol{F}$, Representative MESI images of cortical blood flow. Example ROls for each region type are indicated: $P$, Parenchymal $(\mathbf{G}) ; A$, artery/arteriole $(\boldsymbol{H}) ; \boldsymbol{V}$, vein/venule $(\boldsymbol{I})$. $\mathbf{G}-\boldsymbol{I}$, Correlations of regional blood flow between defined ROls over the 3 month interval $(N=6$ mice, $N=8$ ROls per region type per mouse). Diagonal dashed lines indicate the line of equality. Cortical blood flow was highly consistent within defined regions across the interval.

or $30 \mathrm{~d}$ after permanent middle-cerebral artery occlusion relative to brain endothelial cells in sham-operated mice (Munji et al., 2019). Differentially expressed genes were defined as $\log _{2}$ (fold change) $>1$ or $<-1$ and FDR-adjusted $p<0.05$. Metascape was used to evaluate gene ontology enrichment in upregulated genes (Zhou et al., 2019). Gene sets related to "angiogenesis" and "extracellular matrix organization" ontology terms were derived from GO:0001568 and R-HSA-1474244 gene ontology identifiers, respectively. 
A

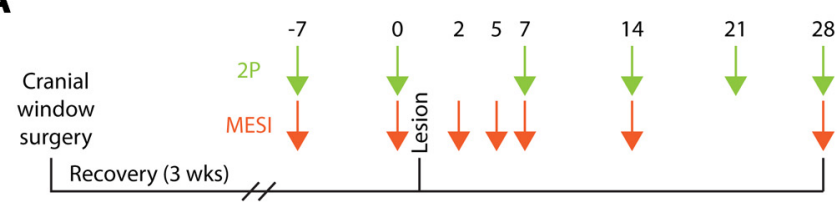

B

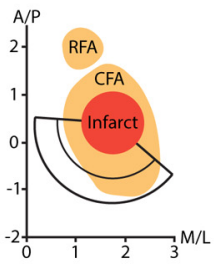

C Pre-infarct
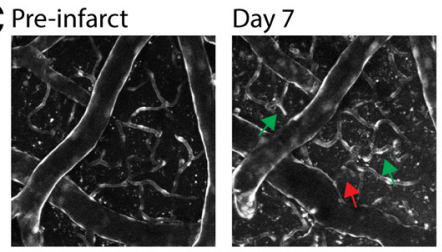

Tie2-GFP
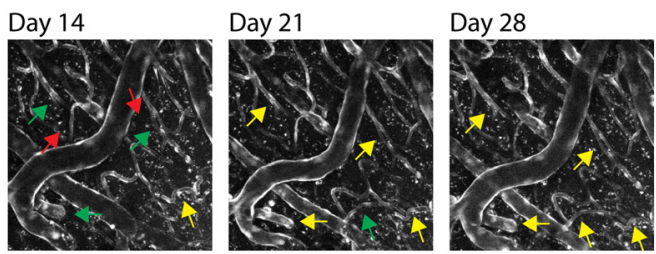

Persistent

D
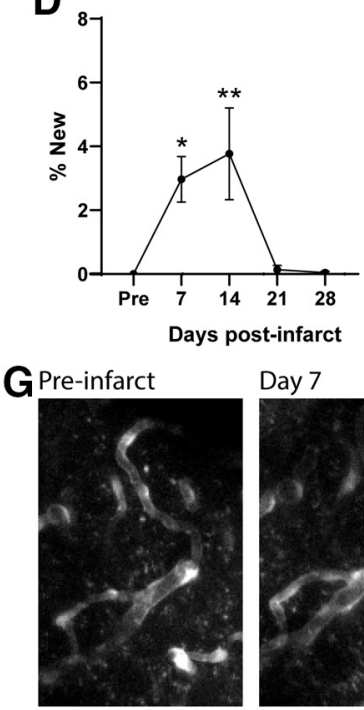

Day 7

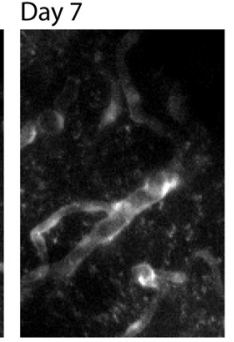

E

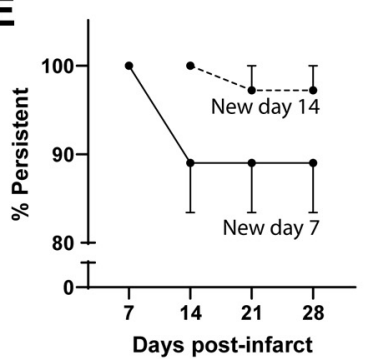

Day 14

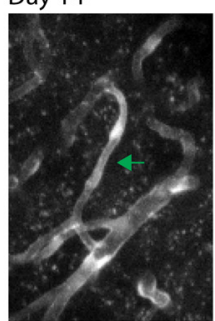

Day 21

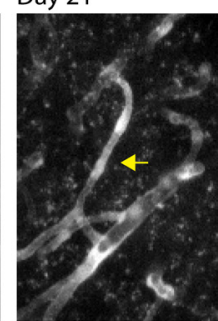

$\mathbf{F}$

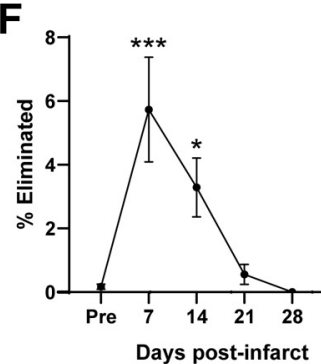

Days post-infarct

Day 28

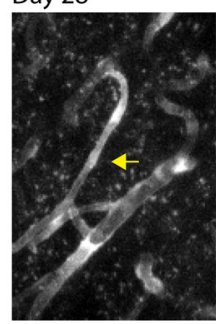

$50 \mu \mathrm{m}$
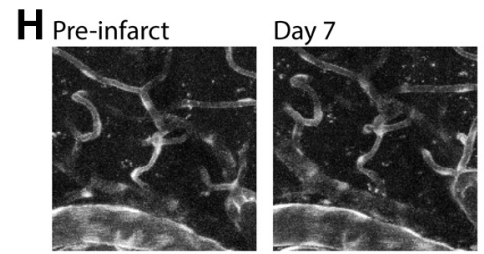

Day 14

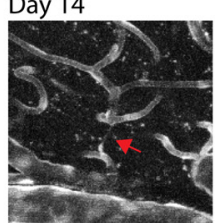

Day 21
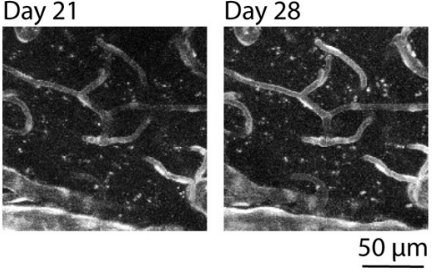

I
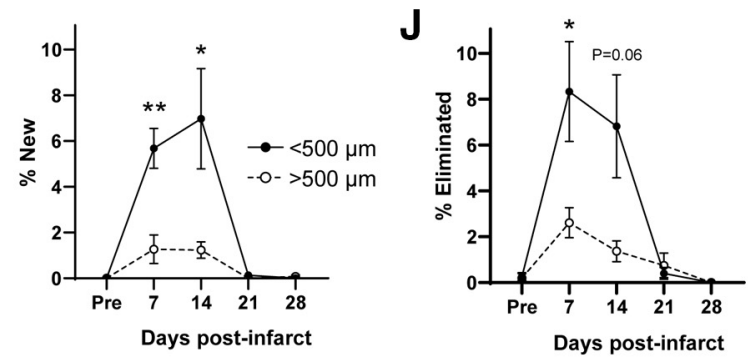

Figure 5. In vivo $2 \mathrm{P}$ time-lapse imaging reveals a spatially graded, temporally restricted window of vascular plasticity after stroke. A, Timeline for multimodal imaging of vascular structure and blood flow in Tie2-GFP mice $(N=6)$. B, Diagram indicating approximate 2P imaged regions relative to the infarct and rostral (RFA) and caudal (CFA) forelimb areas of motor cortex. Numbers indicate millimeters relative to bregma. C, 2 P time-lapse images showing examples of vessel elimination, formation, and new vessel persistence. There was a period of heightened structural vascular plasticity in peri-infarct cortex during the first 2 weeks after infarct characterized by increases in capillary formation $(\boldsymbol{D})$ and elimination $(\boldsymbol{F}) .{ }^{*} p<0.05 ;{ }^{* *} p<0.01$; ${ }^{* * *} p<0.001$; versus pre-infarct (Holm-Sidak tests). $\boldsymbol{E}$, Persistence of new segments. More than $90 \%$ of new capillaries formed after infarct persisted to $28 \mathrm{~d}$ after infarct. All new capillaries that survived to the next imaging time point survived to day 28. $\boldsymbol{G}$, High-magnification example of formation and persistence of a new capillary segment. $\boldsymbol{H}$, High-magnification example of elimination of a single capillary segment. The magnitude of structural vascular plasticity was spatially graded.
Statistical analysis. Statistical analysis was done using GraphPad Prism version 8.4. Two-tailed $t$ tests, Mann-Whitney $U$ tests, one- or two-way ordinary or repeated-measures ANOVAs, and linear regressions were used as noted in the text and as appropriate. Independent means with normally distributed data and similar variance were compared with two-tailed $t$ tests. Variance was compared with $F$ tests or Brown-Forsythe tests. Mann-Whitney $U$ tests were used when data were not normally distributed. Significant ANOVAs were followed by post hoc Holm-Sidak corrected multiple comparisons. $\alpha$ was set at 0.05 . Data are presented as mean \pm SEM.

\section{Results}

Vascular structure and cortical blood flow are largely stable in the intact adult brain

All experiments used mice with chronic cranial windows implanted over motor cortex. We used in vivo $2 \mathrm{P}$ imaging to visualize vascular structure in the uppermost $300 \mu \mathrm{m}$ of cortex in Tie2-GFP mice, which express GFP in endothelial cells (Fig. 1). While intravascular dye (Texas Red-conjugated dextran) provided somewhat better signal-to-background than Tie2-driven GFP expression (Fig. 2), a small proportion of capillaries $(0.09 \pm 0.03 \%$, excluding those with a pinched appearance) were only partially filled or completely lacked the intravascular label (Fig. 3). Therefore, time-lapse imaging that relies on intravascular dyes may give a false appearance of changes in perfusion status as changes in vascular structure. We never observed vessels labeled with intravascular dye that did not express GFP. Furthermore, leakage of intravascular dyes can obscure images after stroke when vascular permeability is increased (Schrandt et al., 2015). Therefore, Tie2-GFP mice provide a reliable way to visualize vascular structure independent of momentary perfusion status and without undesirable dye leakage. To map cortical blood flow, we used MESI, a quantitative, label-free optical method suitable for longitudinal measurements (Kazmi et al., 2013; Schrandt et al., 2015; Clark et al., 2019b; He et al., 2020) (Fig. 1F,G). Combining these imaging modalities in the same animals over weeks to months allowed us to monitor and compare changes in

The rates of capillary formation $(\boldsymbol{I})$ and elimination $(\boldsymbol{I})$ were significantly greater in regions within $500 \mu \mathrm{m}$ of the infarct border compared with those further. ${ }^{*} p<0.05$; ${ }^{* *} p<0.01$; between distance bins ( $t$ tests). 


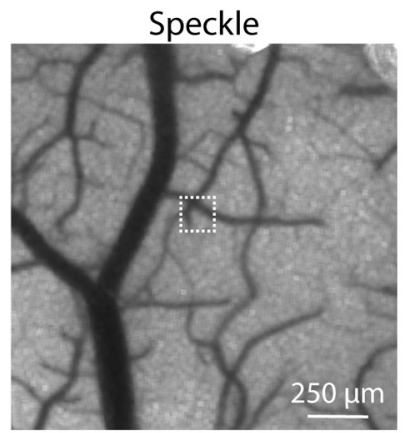

Day 21

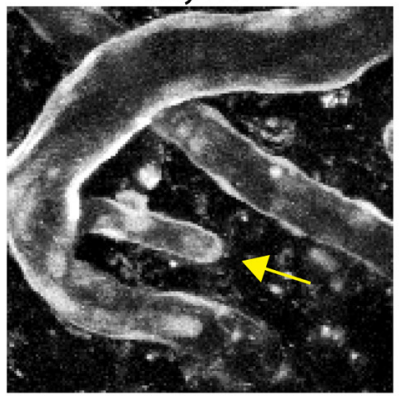

New
Pre-infarct

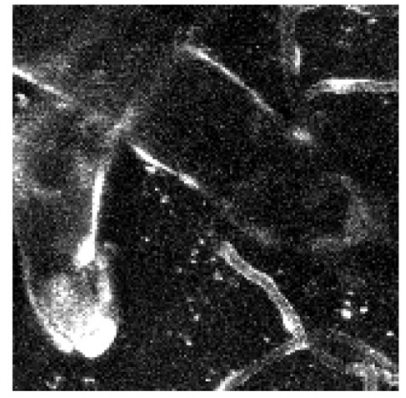

Day 28

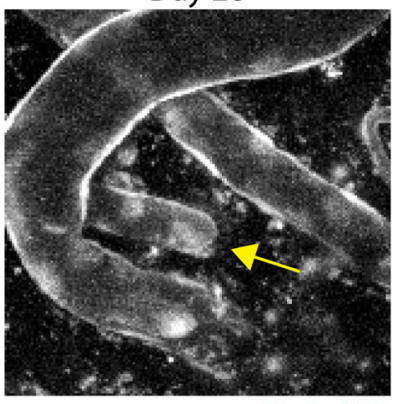

Tie2-GFP

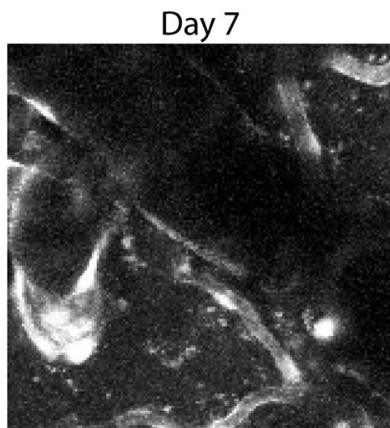

Day 28

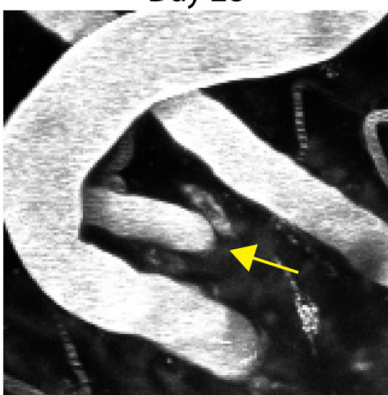

Texas Red

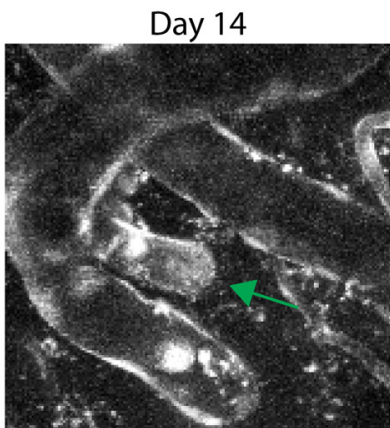

Day 28

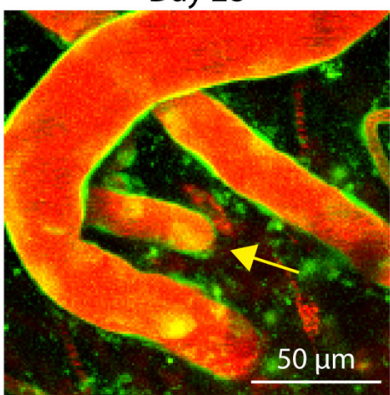

Merge

Persistent

Figure 6. Formation of a new penetrating arteriole after stroke. White box on the speckle contrast image represents the region of $2 \mathrm{P}$ images. The new arteriole segment was visible on day 14 (green arrow) and subsequent imaging time points (yellow arrows).

vascular structure and blood flow with high spatiotemporal precision.

First, we assessed the stability of vascular structure and blood flow in intact cortex. We imaged vascular structure and cortical blood flow in Tie2-GFP mice $(N=6)$ twice with a 3 month interval between imaging sessions (Fig. 4A). We chose this somewhat long interval because of the expected rarity of vascular structural changes in the intact adult brain (Harb et al., 2013). Consistent with previous studies (Harb et al., 2013; Reeson et al., 2018), we observed relatively little structural plasticity (Fig. 4B-E). We observed both infrequent formation $(\sim 0.2 \%$ of all capillaries) and elimination $(\sim 1.4 \%)$ of capillary segments (3572 unique capillary segments were analyzed; Fig. $4 E$ ). This observation of net capillary loss is in line with estimates of age-related vascular rarefaction in mouse cortex (Reeson et al., 2018). We did not observe any gain or loss of arterioles or venules.

Cortical blood flow was also stable in the intact brain (Fig. $4 F)$. There was a $1.7 \pm 2.7 \%$ change in mean parenchymal blood flow over the 3 month interval. Flow in pial arterioles $(-1.3 \pm$ $2.3 \%$ change) and venules ( $1.3 \pm 2.2 \%$ change $)$ was similarly stable. In order to evaluate the magnitude of change within defined regions, we randomly selected 8 ROIs per vessel type (parenchymal, artery/arteriole, and vein/venule) per animal and computed the correlation coefficient of regional blood flow (quantified as ICT) (Kazmi et al., 2013; Clark et al., 2019b) between the two imaging time points. Regional blood flow was highly consistent between imaging time points in all vessel types (Fig. 4G-I). Overall, these data indicate that vascular structure and blood flow are largely stable over months in the intact adult cortex. Moreover, they establish the utility of our multimodal imaging approach.

\section{Cortical infarcts produce a window of heightened vascular structural plasticity}

To track poststroke vascular remodeling and blood flow in the same animals, we repeatedly imaged young adult Tie2-GFP mice $(N=6)$ before and after cortical infarcts induced by photothrombosis (Figs. $1 D, E, 5 A, B$ ). Infarcts were targeted to the caudal forelimb area of motor cortex. Imaging analyses focused on surrounding peri-infarct cortex in which we expected substantial changes in blood flow and where structural and functional remodeling of multiple cell types is associated with behavioral recovery (Kim et al., 2018; Clark et al., 2019a,b). We 2P imaged vascular structure weekly out to 4 weeks after infarct (Fig. $5 \mathrm{~A}$ ). MESI was performed on the same days as $2 \mathrm{P}$ imaging, except for 3 weeks after infarct, when we expected to see little blood flow change based on previous studies (Schrandt et al., 2015; Clark et al., 2019b) as well as unpublished data. In addition, MESI was performed on days 2 and 5 to capture rapid changes in periinfarct blood flow soon after stroke (Schrandt et al., 2015; Clark et al., 2019b).

We tracked vascular structure in the same regions before and after infarcts were produced, analyzing a total of 1275 unique capillary segments. At least three regions per mouse were $2 \mathrm{P}$ imaged before stroke in a manner that yielded sampling regions within and further than $500 \mu \mathrm{m}$ from the infarct border after stroke (except for one mouse in which we were unable to evaluate a region $>500 \mu \mathrm{m}$ from the lesion border) (Fig. $5 B$ ). Infarcts caused a large increase in capillary plasticity, including loss (oneway repeated measures ANOVA, $F_{(4,20)}=10.11, p=0.0001$ ), formation (one-way repeated measures ANOVA, $F_{(4,20)}=8.27$, $p=0.0004$ ), and selective stabilization (persistence) of new segments (Fig. $5 C-H$ ). Importantly, this structural plasticity was 


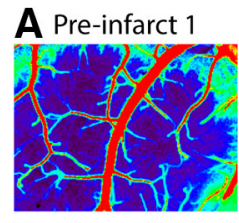

B

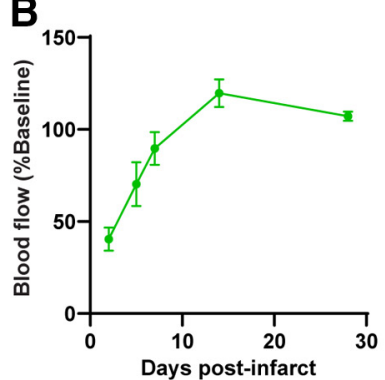

F

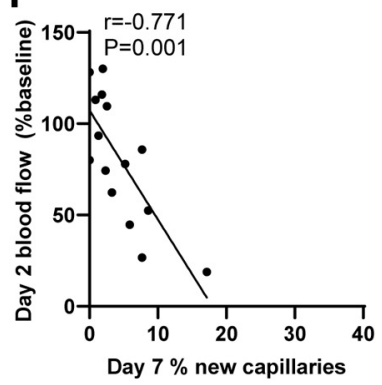

Pre-infarct 2

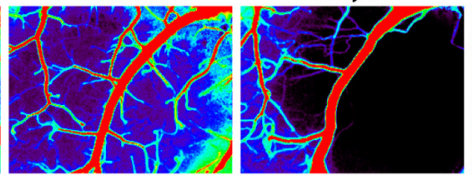

C

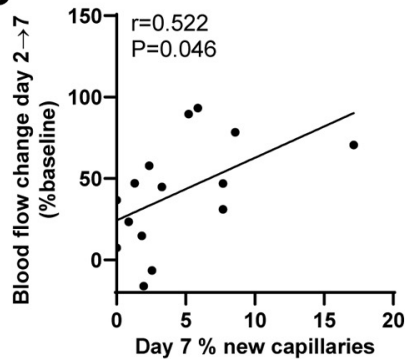

G

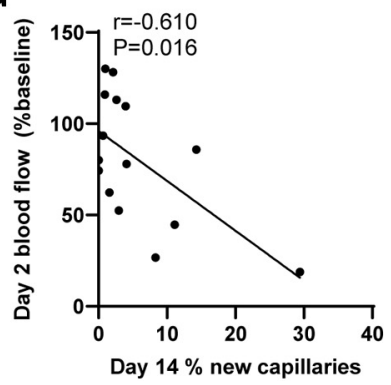

Day 7

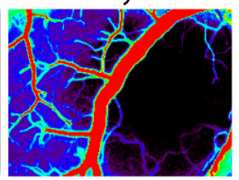

D

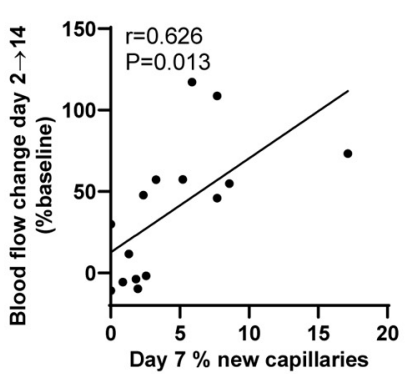

H

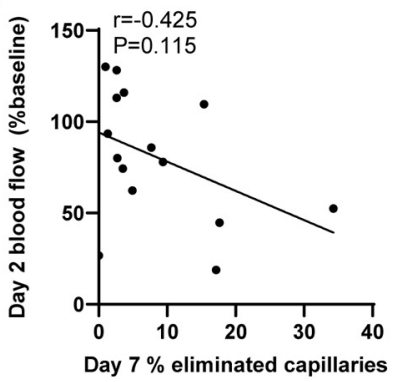

Day 14

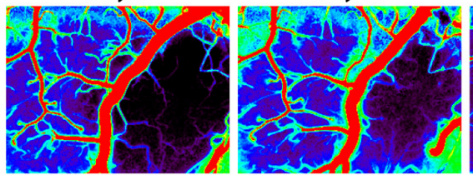

E

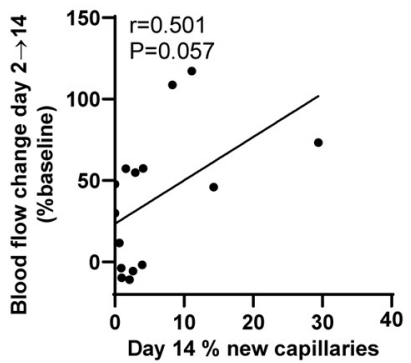

\section{I}

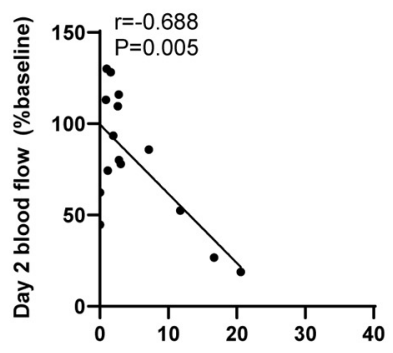

Day $14 \%$ eliminated capillaries

Figure 7. Microvascular plasticity is associated with recovery of peri-infarct blood flow. $\boldsymbol{A}$, Time-lapse MESI of cortical blood flow before and after photothrombosis. $\boldsymbol{B}$, Longitudinal measurements of peri-infarct blood flow after stroke. Sampled regions were within $\sim 500 \mu \mathrm{m}$ of the infarct border. Restoration of blood flow was coincident with the period of heightened structural vascular plasticity. C-E, Local capillary formation during the peak of plasticity was correlated with increases in blood flow within the same region over several time scales. $F-I$, Acute blood flow deficits (day 2 after infarct) predicted the magnitude of later regional capillary plasticity. Changes in blood flow are presented as the difference in blood flow, expressed as a percentage of baseline blood flow, between the indicated time points.

restricted to the first 2 weeks after infarct; later time points showed minimal levels of change similar to those before the infarct (days 7 and 14 vs pre-infarct, Holm-Sidak test, $t_{(20)} \geq$ $2.806, p \leq 0.0324)$. Among newly formed capillary segments, $>90 \%$ were maintained throughout the remaining imaging sessions (Fig. 5E). All new segments that survived at least 1 week persisted throughout the remaining imaging sessions. Analysis of vascular plasticity by distance from the infarct border revealed that stroke-induced plasticity was spatially graded: the relative magnitude of change in regions $>500 \mu \mathrm{m}$ from the infarct border was $\sim 25 \%$ relative to those within $500 \mu \mathrm{m}$ (Fig. 5I,J).

We also observed one possible case of formation of a new penetrating arteriole (Fig. 6), but it did not have any discernible lower-order branches in the region imaged. We cannot completely rule out the possibility that this arteriole came into view because of significant tissue shifting, although that seems unlikely given that lack of extreme displacement in surrounding vessels. The relative lack of formation of new arterioles and venules indicates that capillary remodeling is the predominant mode of poststroke structural vascular plasticity.

Together, these data reveal that focal infarcts create a temporally restricted window of heightened microvascular plasticity in peri-infarct cortex in a spatially graded manner. They also reveal that the formation of new vascular segments is a somewhat conservative process; the number of new capillary segments was small relative to the stable network, and few new segments were formed that did not persist to subsequent imaging sessions.
Structural vascular plasticity is associated with the reestablishment of peri-infarct blood flow

Having established the spatiotemporal dynamics of poststroke vascular structural plasticity, we next assessed its relationship with blood flow. Repeated MESI revealed a delayed recovery of periinfarct (within $\sim 500 \mu \mathrm{m}$ from the infarct border) parenchymal blood flow by approximately day 7 after infarct. Following a mild hyperemia at day 14 , blood flow returned to nearly pre-infarct levels at day 28 (Fig. $7 A, B$ ). This reestablishment of blood flow was temporally coincident with the window of structural vascular plasticity. We then measured parenchymal blood flow in the same regions in which vascular structure was repeatedly imaged. Importantly, within discrete regions, high rates of new capillary formation were significantly correlated with the reestablishment of parenchymal blood flow (Fig. 7C-E). Therefore, regional vascular capillary formation is associated with local changes in blood flow. This finding is consistent with the idea that vascular structural plasticity supports the reestablishment of peri-infarct blood flow.

If structural vascular plasticity is an adaptive response to ischemia, we would hypothesize that the severity of ischemia would predict the amount of plasticity. Correlations between the relative blood flow $2 \mathrm{~d}$ after infarct and the magnitude of capillary formation and elimination measured at days 7 and 14 revealed that the initial severity of regional ischemia was indeed significantly correlated with the amount of new and eliminated capillary segments during the peak in plasticity (Fig. $7 F-I$ ). The variability in initial severity of ischemia across discrete regions likely reflects differences in the ability for residual vascular pathways to compensate for reduced flow. The variability in capillary formation rates is likely 
A
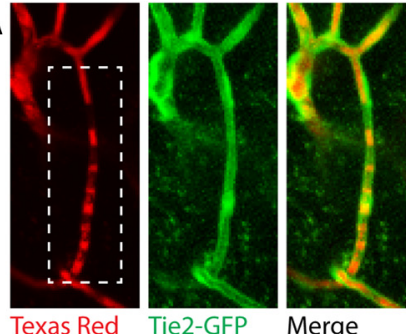

Texas Red

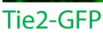

Merge

Stalled - day 28 post-infarct

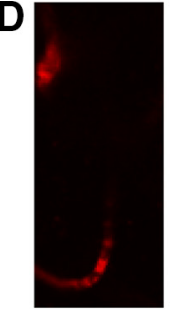

$0 \mathrm{~s}$

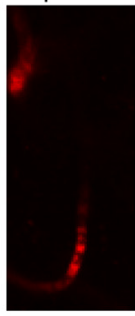

$0 \mu \mathrm{m}$

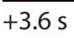

$-4 \mu \mathrm{m}$
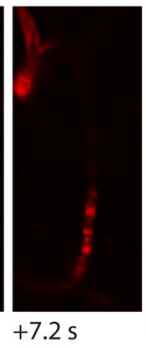

$-8 \mu \mathrm{m}$

Flowing

E
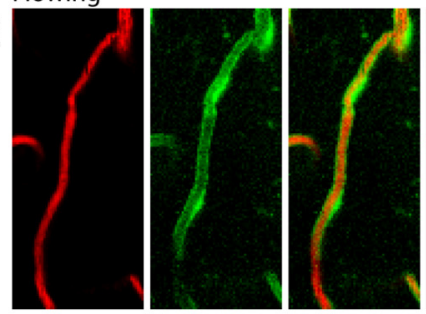

Texas Red

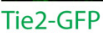

Merge

Stalled - naive
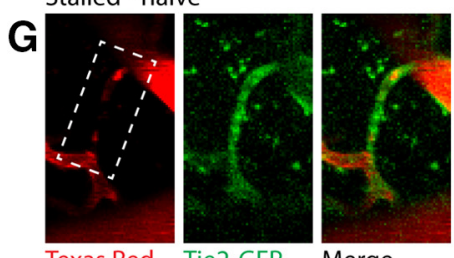

Texas Red Tie2-GFP

Merge

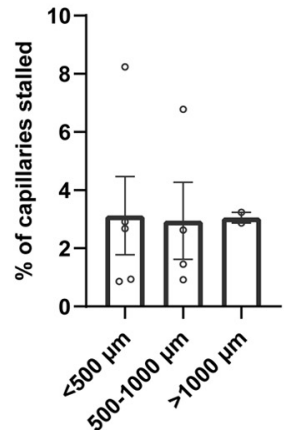

Distance from infarct border
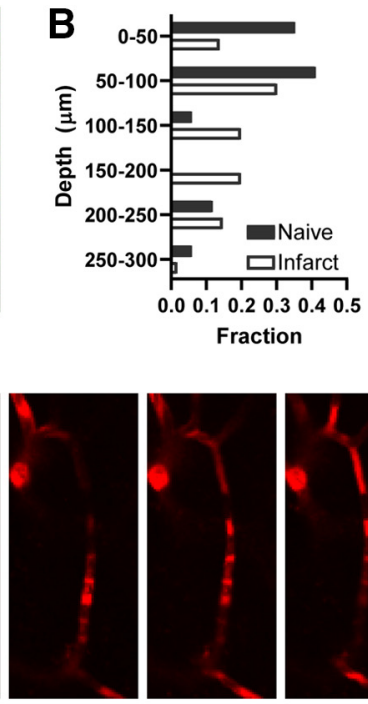

$+10.8 \mathrm{~s}$

$-12 \mu \mathrm{m}$

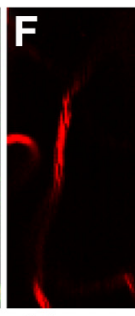

$+3.6 \mathrm{~s}$

$-4 \mu \mathrm{m}$

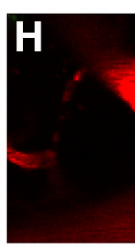

$+3.6 \mathrm{~s}$ $-4 \mu \mathrm{m}$ dis

J

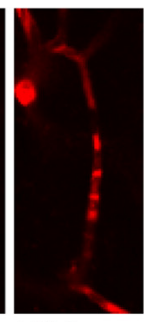

$+14.4 \mathrm{~s}$ $-16 \mu \mathrm{m}$
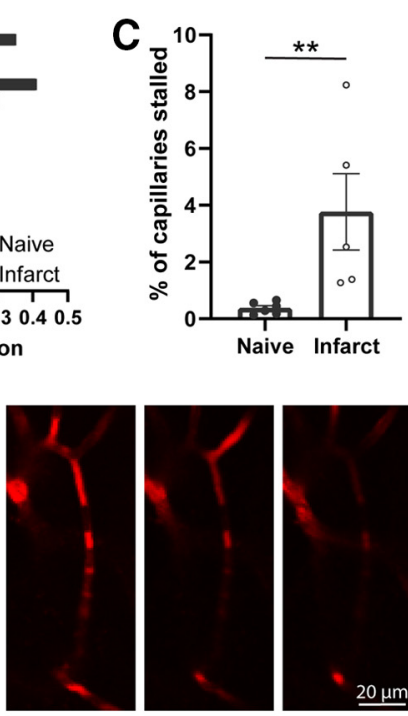

$+18 \mathrm{~s}$ $-20 \mu \mathrm{m}$

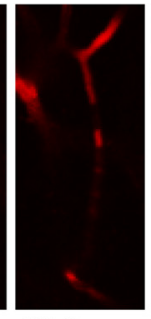

$+21.6 \mathrm{~s}$ $-24 \mu \mathrm{m}$
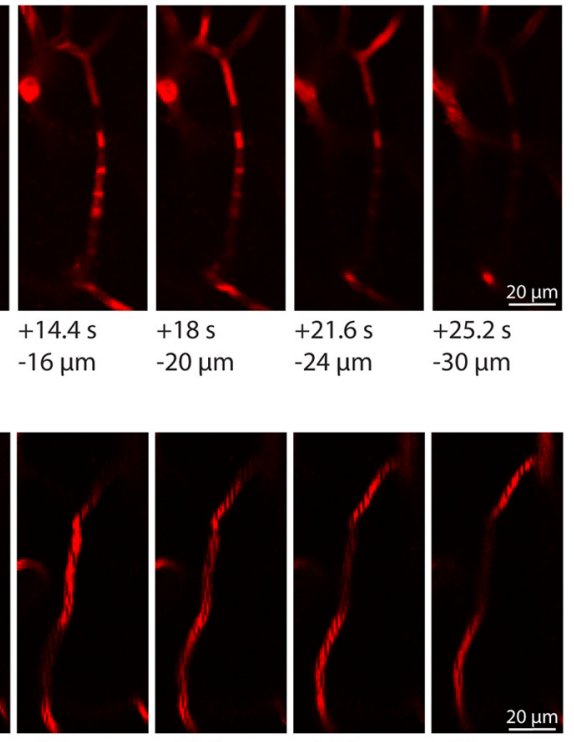

$+10.8 \mathrm{~s}$ $-12 \mu \mathrm{m}$

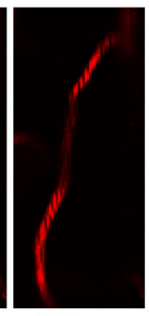

$+14.4 \mathrm{~s}$ $-16 \mu \mathrm{m}$ $-8 \mu \mathrm{m}$

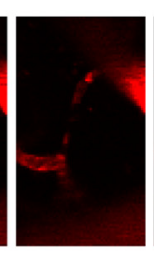

$+5.4 \mathrm{~s}$ $-6 \mu \mathrm{m}$ $6 \mu$

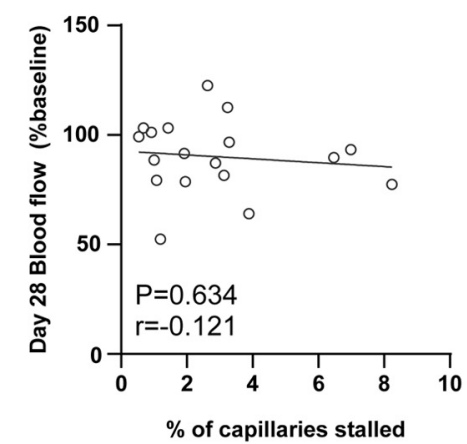

Figure 8. Stroke increases the frequency of capillary stalls in peri-infarct cortex. Stalled capillaries were identified by an absence of blood cell movement in 2P images. $\boldsymbol{A}$, Example of a stalled capillary in peri-infarct cortex $28 \mathrm{~d}$ after stroke (dashed box). Images are maximum projection images. $\boldsymbol{B}$, Depth distribution of stalled capillaries summed across mice. In naive mice, stalled capillaries tended to be located within the superficial $100 \mu \mathrm{m}$ of cortex. At $28 \mathrm{~d}$ after stroke, stalled capillaries were observed more evenly throughout the imaged depth of cortex. C, Proportion of stalled capillaries in naive mice compared with $28 \mathrm{~d}$ after infarct. Stalled capillaries were $\sim 3-20$ times more frequent after stroke relative to naive mice. ${ }^{* *} p<0.01$ (Mann-Whitney $\boldsymbol{U}$ test). $\boldsymbol{D}$, Time-lapse imaging of the stalled capillary in $\boldsymbol{A}$ through $z$ steps. Note the immobile bright (labeled plasma) and dark (plasma excluded) regions. $\boldsymbol{E}$, Example of a flowing capillary (maximum projection images). $\boldsymbol{F}$, Timelapse imaging of the capillary in $\boldsymbol{E}$ through $\boldsymbol{z}$ steps. Narrow dark streaks represent blood cell movement. $\boldsymbol{G}$, Example of a stalled capillary in cortex of a naive, uninjured mouse (dashed box). Images are maximum projection images. $\boldsymbol{H}$, Time-lapse because of both the severity of ischemia and the effectiveness of new capillaries in restoring blood flow.

\section{Capillary stalls are frequent in peri- infarct cortex}

Previous studies have reported spontaneous stalls in capillary blood cell flux (i.e., lack of flow) (Villringer et al., 1994; Reeson et al., 2018; Cruz Hernández et al., 2019; Erdener et al., 2019, 2020). Capillary stalls are visible in $2 \mathrm{P}$ raster scanned images by an absence of blood cell streaks in plasma labeled with intravascular dyes (Reeson et al., 2018) (Figs. 8, 9). Capillary stalls are implicated in age-related vascular rarefaction (Reeson et al., 2018; Schager and Brown, 2020), acute postischemic penumbral tissue loss (Erdener et al., 2020), and cognitive impairments in Alzheimer's disease model mice (Cruz Hernández et al., 2019). The duration of stalls is variable in the uninjured mouse cortex: $\sim 0.45 \%$ of cortical capillaries are estimated to be stalled at a given moment (Erdener et al., 2019), and $\sim 0.12 \%$ of capillaries are obstructed for at least 20 min over a $2 \mathrm{~h}$ period (Reeson et al., 2018).

Serendipitously, we noticed that stroke dramatically increased the incidence of capillary stalls in peri-infarct cortex at $28 \mathrm{~d}$ after infarct (Fig. 8). Intravascular Texas Red was administered during the $2 \mathrm{P}$ imaging session $28 \mathrm{~d}$ after stroke in 5 of 6 mice. Initially, we had intended to confirm that new, persistent capillary segments formed after infarct were perfused. At least at day 28, all new, persistent capillaries had blood flow, as indicated by a lumen filled with Texas Red. Surprisingly, we noticed large numbers of capillaries lacking blood cell flux: $3.8 \pm 1.3 \%$ of all capillaries were stalled in peri-infarct cortex (4360 capillaries counted across 5 animals; Fig. $8 A-E$ ). In contrast, in naive mice, we observed that $0.38 \pm 0.09 \%$ of all capillaries were stalled (4788 capillaries counted across

\section{$\leftarrow$}

imaging of the stalled capillary in $\boldsymbol{G}$ through $\boldsymbol{z}$ steps. $\boldsymbol{I}$, Percent of capillaries that were stalled in peri-infarct cortex $28 \mathrm{~d}$ after infarct by distance from the infarct border. Each point represents a single animal for the indicated distance bin. Distances $>500 \mu \mathrm{m}$ could only be analyzed in a subset of animals. There was no effect of distance on the incidence of capillary stalls (one-way ANOVA, $F_{(2,8)}=0.005$, $p=0.995)$. J, Scatter plot of the incidence of capillary stalls in each $2 \mathrm{P}$ imaged region and day 28 parenchymal blood flow measured in the same region ( $N=18$ regions; each point represents a single region) from $N=5$ mice. The incidence of capillary stalls did not predict regional blood flow, indicating that any effect of stalls on blood flow was highly localized. 
A

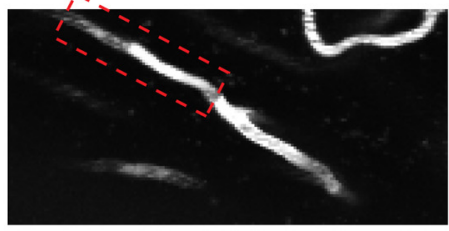

B
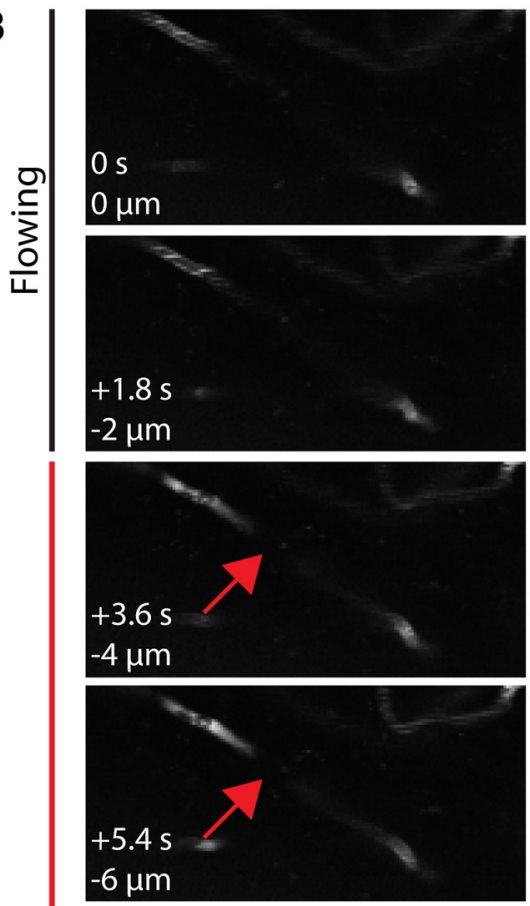

$\frac{\bar{d}}{\stackrel{\bar{\Xi}}{\sim}}$
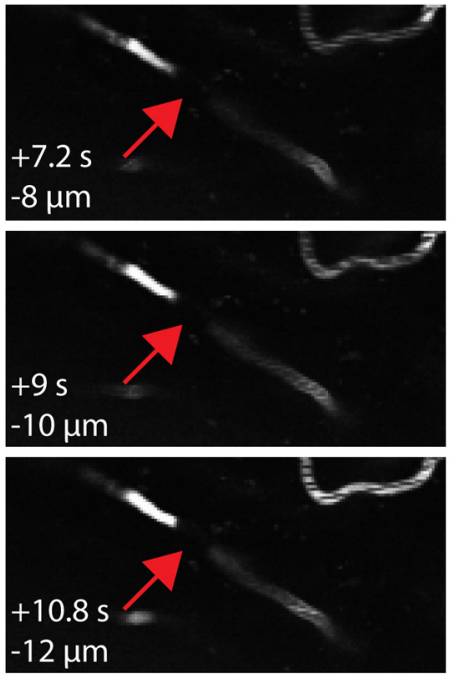

\section{个 obstruction}
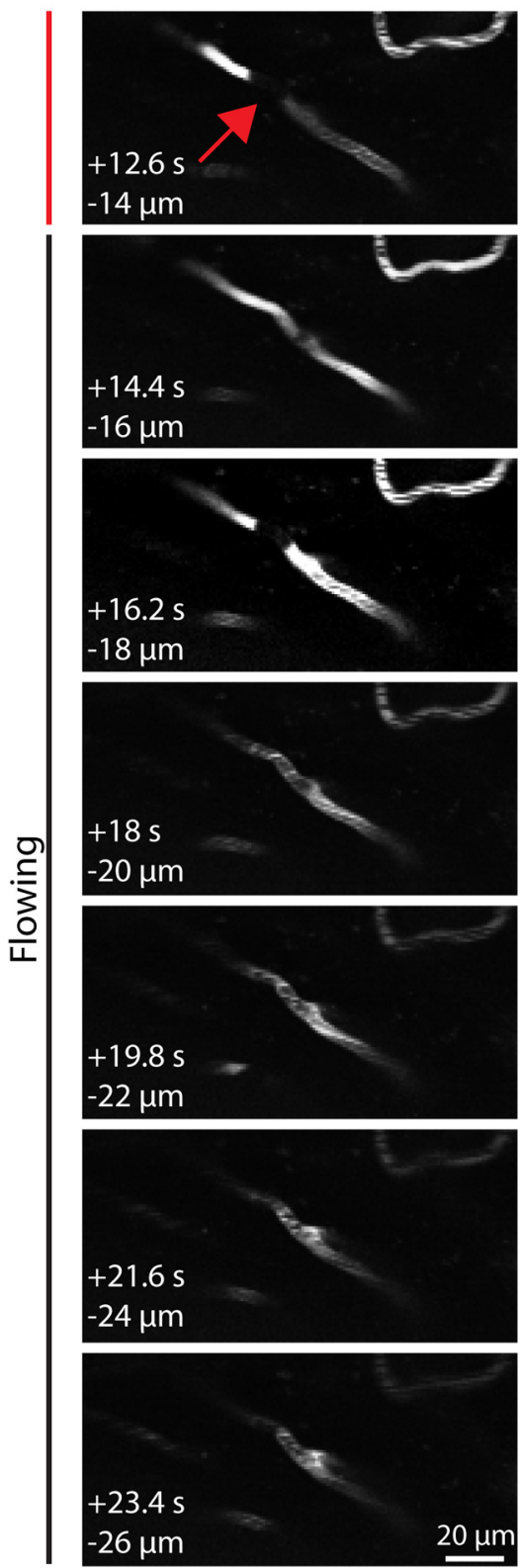

Figure 9. Example of a transiently stalled capillary. $\boldsymbol{A}$, Maximum intensity projection of a capillary that was transiently stalled. Red box represents the segment that was stalled. $\boldsymbol{B}$, Time-lapse images of the transiently stalled capillary in $\boldsymbol{A}$, through $2 \mu \mathrm{m} z$ steps. For comparison, streaks of moving blood cells are visible in all images in the capillary at the top right. Red arrow indicates an obstruction.

6 animals; Fig. 8G), consistent with other studies in intact cortex (Reeson et al., 2018; Erdener et al., 2019). Therefore, the incidence of capillary stalls was on average 10 -fold higher at $28 \mathrm{~d}$ after infarct compared with naive mice (Fig. $8 C$; Mann-Whitney $U$ test, $U=0, p=0.004)$. Capillary stalls were widespread throughout peri-infarct cortex. There was no difference in the proportion of capillaries stalled with distance from the infarct border as measured out to $1500 \mu \mathrm{m}$ (Fig. 8I).
The majority of capillary stalls in naive mice were in the uppermost $100 \mu \mathrm{m}$ of cortex (Fig. 8B), consistent with previous work (Reeson et al., 2018). However, at $28 \mathrm{~d}$ after stroke, stalls were somewhat more evenly distributed throughout the depth of imaged cortex; $3.2 \%$ of capillaries formed after stroke were stalled on day 28 , which is in line with the overall incidence of stalls, indicating similar incidence in new and preexisting vessels. The regional incidence of capillary stalls at $28 \mathrm{~d}$ after infarct was not correlated with parenchymal blood flow (Fig. 8J). Therefore, we suspect that the impact of stalls on blood flow and oxygen availability is highly localized (Erdener et al., 2020). When blood flow was stalled, there were typically either several stationary blood cells visible along the length of the vessel segment (e.g., Fig. 8A) or large lengths of the segment lacking Texas Red (e.g., Fig. 8G). Therefore, we were unable to quantify the precise location of stalls along individual capillary segments.

In 3 cases, we observed transiently stalled capillaries (i.e., blood cells were immobile for only part of the time the segment was in view; Fig. 9). These cases demonstrate that stalls are dynamic and can last $<10 \mathrm{~s}$, as previously reported (Erdener et al., 2019, 2020). Furthermore, since almost all stalled capillaries were stalled for the entire time they were visible, stalls generally persist for $>10 \mathrm{~s}$. In summary, these data indicate that capillary stalls, which represent a deficit in microvascular hemodynamics, are surprisingly common in peri-infarct cortex, even $28 \mathrm{~d}$ after the induction of ischemia. Therefore, highly localized perturbations in capillary blood flow persist for at least weeks after stroke despite substantial restoration of regional blood flow to the majority of periinfarct cortex.

\section{The reestablishment of peri-infarct} blood flow is associated with behavioral recovery

In order to examine how the reestablishment of peri-infarct blood flow is related to recovery of motor function, we repeatedly imaged blood flow with MESI and measured performance on the single-seed reaching task before and after photothrombotic infarcts were induced in forelimb motor cortex of C57BL/6J mice (Clark et al., 2019a,b) (final $N=10$ ) (Fig. 10A). The single-seed reaching task is a highly sensitive measure of skilled forelimb use that is analogous to human reaching (Klein et al., 2012). Skilled forelimb use is a translationally relevant behavioral measure because upper limb impairment is the most common poststroke deficit (Lawrence et al., 2001). 


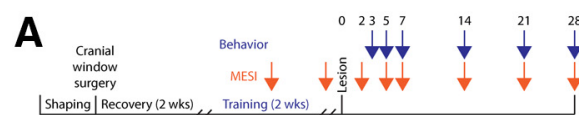

B

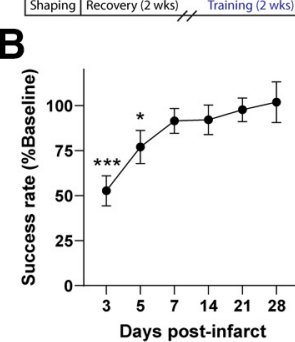

C

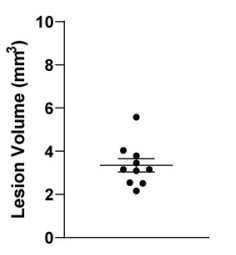

I

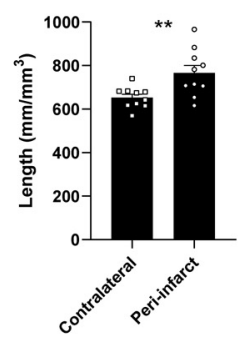

N

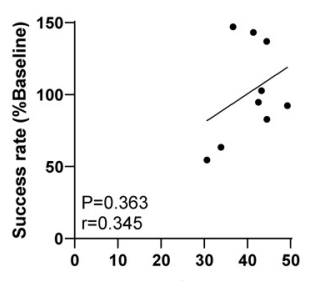

Peri-infarct IB4 ${ }^{+}$Area Fraction

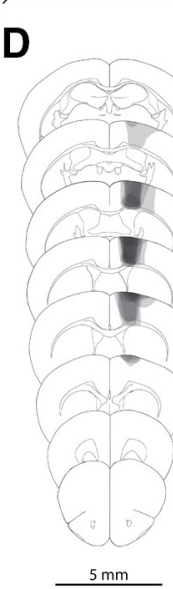

J

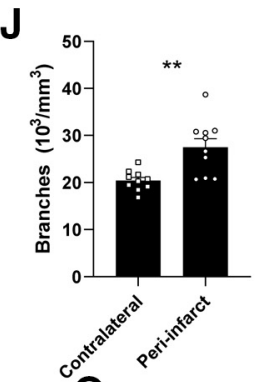

0

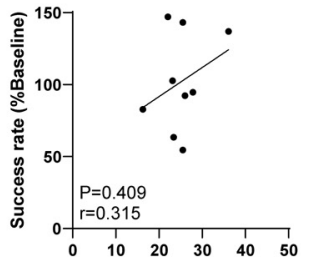

Peri-infarct Tomato Lectin ${ }^{+}$Area Fraction

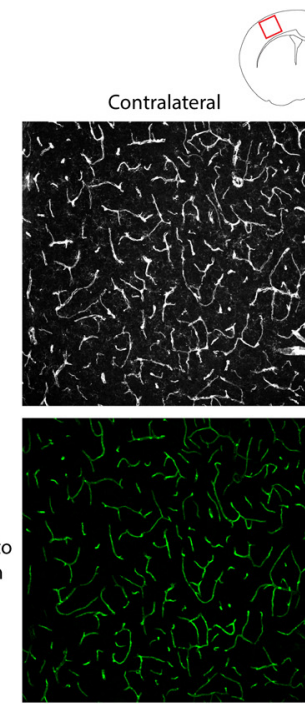

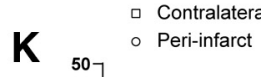

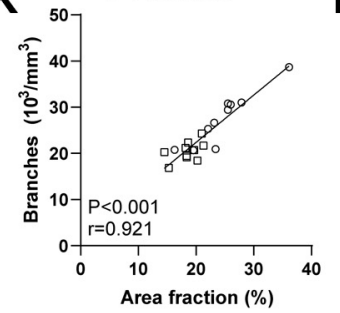

L

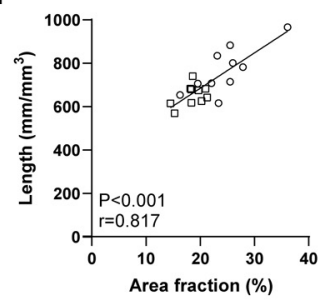

P

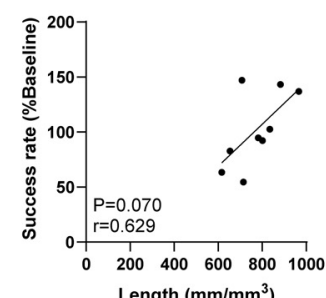

F

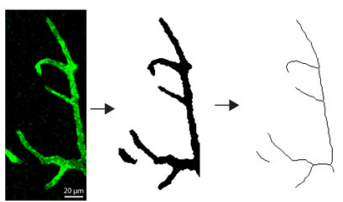

G

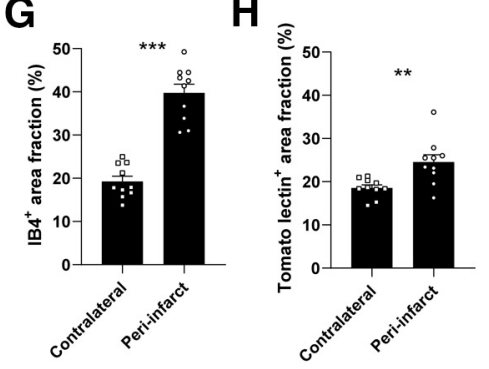

M

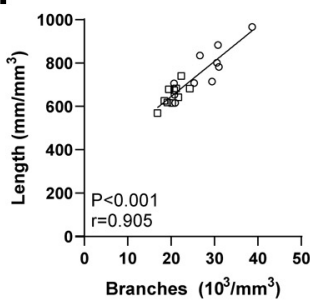

Q

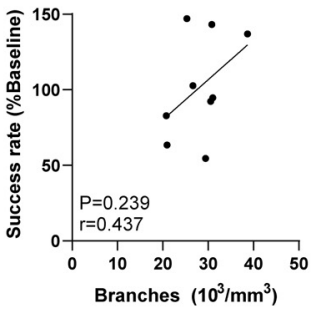

Figure 10. Behavioral deficits and histologic evidence of vascular remodeling after stroke. $A$, Timeline for behavioral training and testing and repeated imaging of blood flow in $(57 B L / 6 \mathrm{~J}$ mice. $\boldsymbol{B}$, Infarcts caused transient deficits on the single-seed reaching task. ${ }^{*} p<0.05$; ${ }^{* * *} p<0.001$; versus day 28 (Holm-Sidak test). $N=9$. C, Lesion volume measurements. $\boldsymbol{D}$, Schematic reconstructions of lesions. Darker regions represent more overlap between animals. $N=10$. $\boldsymbol{E}$, Representative confocal images (maximum intensity projections) of IB4 and tomato lectin labeling of vasculature in peri-infarct and homotopic contralateral cortex. Top, Schematic represents sampled regions (red boxes). $\boldsymbol{F}$, Example of binarization and skeletonization of vessels labeled with tomato lectin. Infarcts increased the area fraction of $\mathrm{IB}^{+}(\boldsymbol{G})$ and tomato lectin ${ }^{+}(\boldsymbol{H})$ vessels in peri-infarct cortex relative to the intact contralateral cortex. ${ }^{* *} p<0.01 ;{ }^{* * *} p<0.001 ;$ contralateral versus peri-infarct ( $t$ tests). Vascular length $(\boldsymbol{I})$ and branch density $(\boldsymbol{l})$, measured from tomato lectin-labeled vessels, were also significantly increased in peri-infarct cortex relative to contralateral cortex. ${ }^{* *} p \leq 0.007, t_{(18)} \geq 3.04$ ( $t$ tests). $\boldsymbol{K}-\boldsymbol{M}$, Area fraction, vascular length, and branch density were all strongly correlated with one another. Measurements are from tomato lectin-labeled vessels. $\mathbf{N}-\mathbf{Q}$, Area fraction, vascular length, and branch density in peri-infarct cortex were positively, but not significantly, correlated with behavioral performance on day 28 after infarct. $\mathbf{0}-\mathbf{Q}$, Measurements from tomato lectin-labeled vessels.

Motor cortical infarcts caused transient deficits on the singleseed task that reached an asymptote of recovery by $7 \mathrm{~d}$ after infarct (Fig. $10 B-D$; repeated measures ANOVA, $F_{(5,40)}=5.531$, $p<0.001$ ). Lesion size, measured at day 28 , was not significantly correlated with behavioral performance at any time point $\left(r_{(1,8)}=\right.$ -0.51 to $0.20, p \geq 0.104$ ). All infarcts mainly occupied the caudal forelimb area of motor cortex (Fig. 10D), as we have previously defined by intracortical microstimulation and cytoarchitecture (Tennant et al., 2011), which contains representations for the digits, wrist, and elbow. Representations for the shoulder, trunk, and hindlimb were likely also affected, but to a lesser extent.

Retro-orbital injections of tomato lectin were given immediately before perfusion to label perfused vessels (Robertson et al.,
2015). We also costained sections with the vascular label isolectin B4 (Wälchli et al., 2015). The area fraction of vessels labeled with both techniques was significantly greater in peri-infarct cortex compared with homotopic contralateral cortex, providing histologic evidence for remodeling of peri-infarct vasculature (Fig. $10 E-H ; t_{(18)} \geq 3.305, p \leq 0.004$ ). Vessel length and branch density, measured from images of tomato lectin-labeled vessels, were also increased in peri-infarct cortex (Fig. 10I,J). Vascular area fraction, vessel length, and branch density were all tightly correlated with one another (Fig. $10 K-M$ ). Histologic measures of peri-infarct vasculature were positively but not significantly correlated with behavioral outcome (Fig. 10N-Q). Histologic measures of vascular morphology likely reflect an 

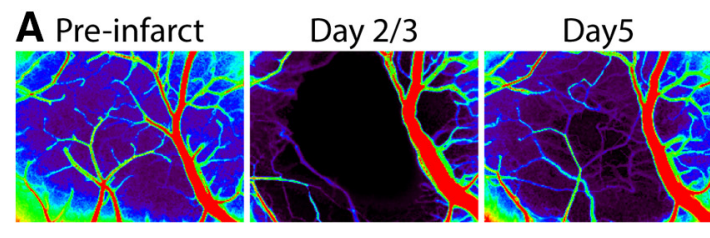

Day 7

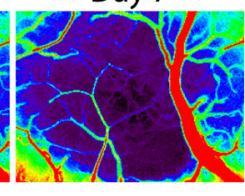

Day14

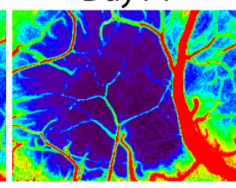

Day 21

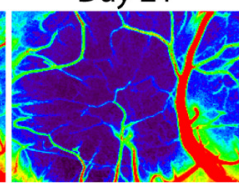

Day 28

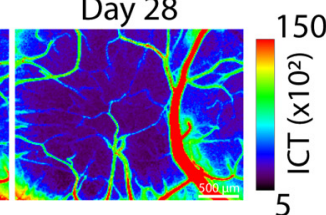

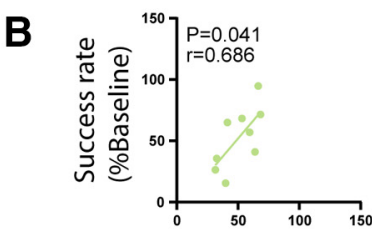
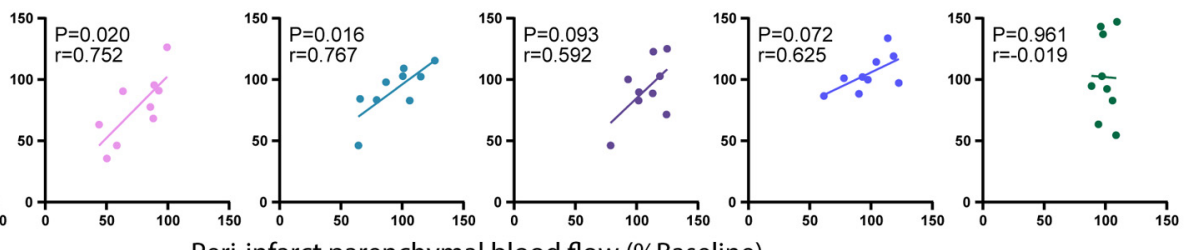

C

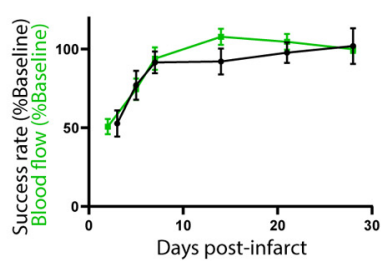

D

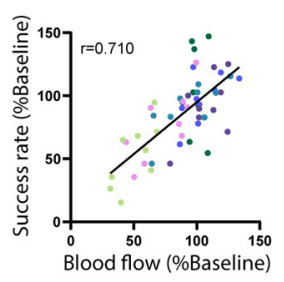

Day post-infarct

- $2 / 3$

- 5

- 7

- 14

21

- 28

Figure 11. Reestablishment of peri-infarct blood flow is associated with recovery of motor function. $\boldsymbol{A}$, Time-lapse MESI of cortical blood flow before and after photothrombosis. $\boldsymbol{B}$, Correlations between peri-infarct parenchymal blood flow and motor performance on the single-seed reaching task by day. Data from each plot are from the time point indicated above in $\boldsymbol{A}$. Blood flow was significantly correlated with motor performance during the first week after infarct. Positive associations on days 14 and 21 did not reach significance. $\boldsymbol{C}$, The time courses of recovery of peri-infarct blood flow and motor function showed a strong temporal association. $\boldsymbol{D}$, Peri-infarct blood flow and behavioral performance were highly correlated across all days. $\boldsymbol{E}$, Acute (day 2) peri-infarct blood flow was not predictive of day 28 motor performance.

aggregate measure of vessel formation, elimination, densitypreserving reorientation (Schrandt et al., 2015; Kirst et al., 2020), and tissue shifting because of collapse of the infarct (Brown et al., 2007). These data indicate that single time point histologic measures of vascular morphology throughout the depth of cortex only weakly predicted spontaneous behavioral recovery. Histologic measures were also not significantly correlated with restoration of peri-infarct blood flow (linear regressions, day $2 \rightarrow 7, r_{(1,8)} \leq 0.16, p \geq 0.651$; day $2 \rightarrow 14, r_{(1,8)} \leq$ $0.39, p \geq 0.271$ ) or day 28 blood flow (linear regression, $r_{(1,8)}$ $\leq 0.43, p \geq 0.219$ ).

The main goal of our final experiment was to evaluate the relationship between the reestablishment of peri-infarct blood flow and recovery of motor function. We took advantage of natural variability in spontaneous motor recovery and peri-infarct blood flow restoration. In Figure 11, we present correlations between peri-infarct blood flow and behavioral performance throughout the $28 \mathrm{~d}$ postinfarct period. During the first week after infarct, daily peri-infarct parenchymal blood flow was strongly positively correlated with behavioral function (Fig. $11 A, B)$. There were nonsignificant tendencies for similar correlations on days 14 and 21 ( $p \leq 0.093$, linear regression). Plotting the time courses of recovery of blood flow and behavioral function revealed a strong temporal association; improvements in motor function were coupled to peri-infarct blood flow (Fig. $11 C$ ). Indeed, across all time points, blood flow was strongly correlated with motor function (Fig. 11D). By contrast, acute (day 2) peri-infarct perfusion deficits were not predictive of later (day 28) motor performance (Fig. 11E), which indicates that motor recovery was not dependent on acute severity of ischemia in peri-infarct cortex, but rather on the reestablishment of more normal blood flow. Together, our findings are consistent with the idea that poststroke plasticity of vasculature structure functions, at least in part, to support restoration of blood flow, which in turn facilitates behavioral recovery.
Transcriptional programs related to vascular remodeling are transiently activated in peri-infarct endothelial cells

Finally, we hypothesized that transient gene expression changes might underlie the temporally restricted period of vascular plasticity we have identified after focal ischemia. To evaluate this hypothesis, we analyzed RNA sequencing data from a resource on the brain endothelial cell transcriptome in health and disease (Munji et al., 2019). Specifically, we assessed changes in gene expression in mouse peri-infarct endothelial cells 3 and $30 \mathrm{~d}$ after middle cerebral artery occlusion relative to sham-operates. These time points represent gene expression during and after the window of heightened vascular plasticity, respectively. We found that the total number of differentially expressed genes was $\sim 10$ fold greater at 3 (546 upregulated, 151 downregulated) versus $30 \mathrm{~d}$ (26 upregulated, 38 downregulated) (Fig. 12A,B). Among upregulated genes, analysis of enriched gene ontology terms showed strong enrichment for terms related to vascular remodeling (e.g., blood vessel development, extracellular matrix organization, cell division) at day 3 , and relatively reduced enrichment for the same terms at day 30 (Fig. 12C). Gene-level analysis of genes related to angiogenesis (Fig. 12D) and extracellular matrix organization (Fig. 12E) revealed higher expression of all relevant genes at day 3 compared with day 30 . Overall, these data provide molecular level evidence to support that stroke creates a temporally restricted window of heightened vascular plasticity.

\section{Discussion}

Our study reveals the spatiotemporal evolution of vascular structural plasticity after stroke. We demonstrated that vascular structure is stable in the uninjured cortex even over months. However, stroke creates a temporally restricted window of heightened vascular remodeling that occurs in a spatially graded manner from the infarct. We found that the extent of vascular structural plasticity predicts the local reestablishment of blood 
flow. In turn, the degree to which periinfarct blood flow is restored predicts recovery of motor function. Our findings support that vascular structural plasticity is a mechanism for the restoration of blood flow in peri-infarct cortex, which facilitates behavioral recovery.

Past studies of vascular remodeling after stroke have largely relied on histologic and other single time point measures. Such measures lack the ability to observe dynamic changes. With timelapse imaging of the same regions of vasculature before and after stroke, we were able to resolve the elimination, formation, and selective stabilization of single capillary segments. The majority of this structural plasticity occurred during the first 2 weeks after stroke. The concurrent elimination and formation of capillary segments we observed are a departure from the stereotypical pattern of initial growth and subsequent pruning that happens during developmental brain angiogenesis (Harb et al., 2013) and developmental and postnatal angiogenesis in other vascular beds (Korn and Augustin, 2015). We speculate that vessel formation is both triggered and limited by ischemia surrounding the infarct core. Moreover, vessel elimination may be driven largely by pruning of occluded vessels (Korn and Augustin, 2015; Reeson et al., 2018; Erdener et al., 2020).

Even small infarcts cause long-lasting deficits in blood flow, metabolism, and neuronal function that extend broadly throughout surrounding peri-infarct tissue (Carmichael et al., 2004; Shih et al., 2013; Schrandt et al., 2015; Summers et al., 2017). Such deficits are implicated in hindered neural repair and disrupted behavioral function (Brown et al., 2009; Mostany et al., 2010; Shih et al., 2013). Consistent with past studies (Mostany et al., 2010; Schrandt et al., 2015; Clark et al., 2019b), we observed a broad reduction in blood flow in residual peri-infarct cortex that resolved on average by $7 \mathrm{~d}$ after stroke. However, ischemia often persisted longer within subregions of peri-infarct cortex. Importantly, the magnitude of capillary structural plasticity was strongly associated with blood flow recovery, indicating that the formation of new microvascular connections is a mechanism underlying reestablishment of blood flow. We also found that the degree to which perfusion deficits in peri-infarct regions were restored predicted behavioral function. These findings are consistent with the hypothesis that reestablishment of peri-infarct blood flow is linked with behavioral improvement. Reestablishment of blood

D
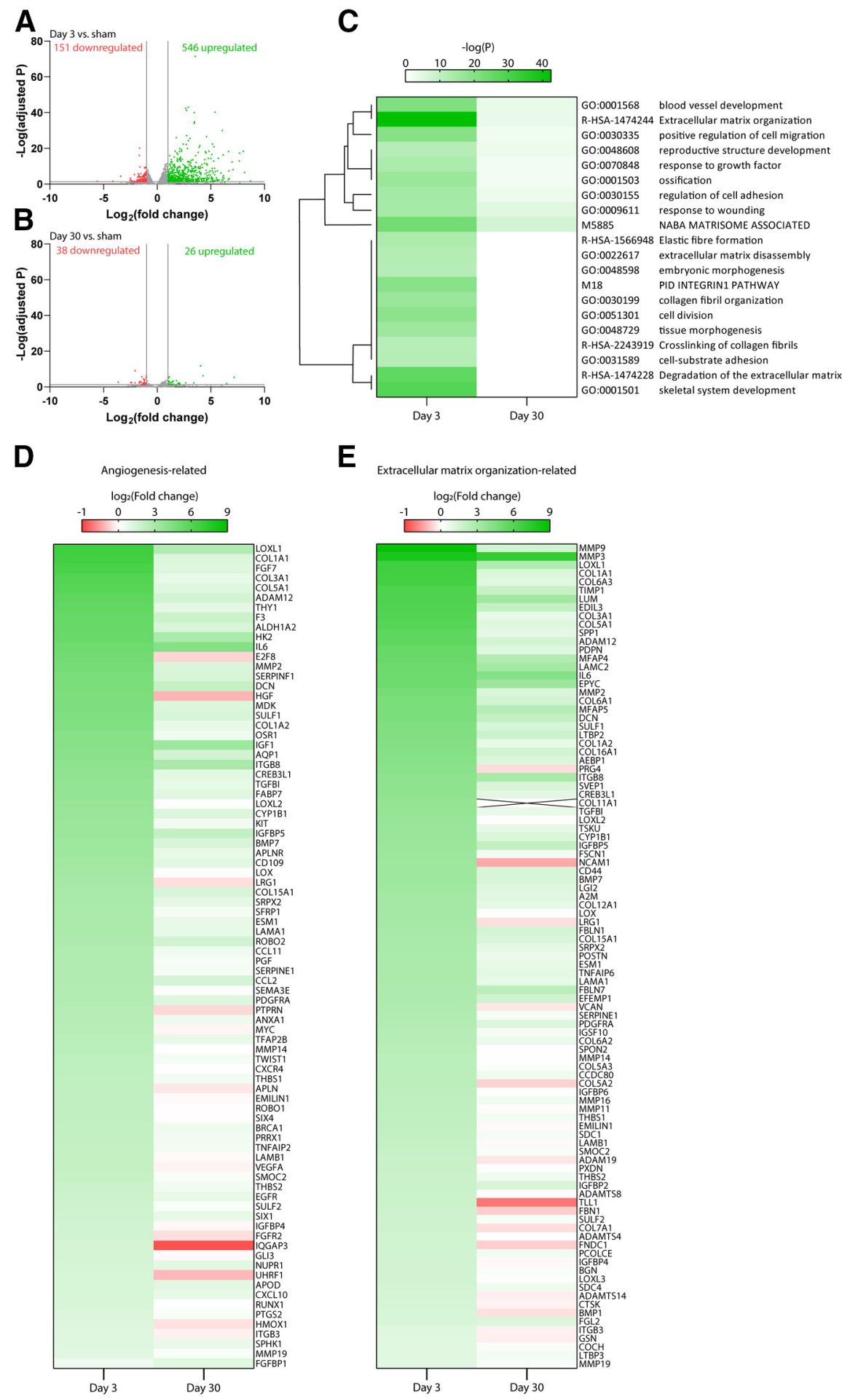

E

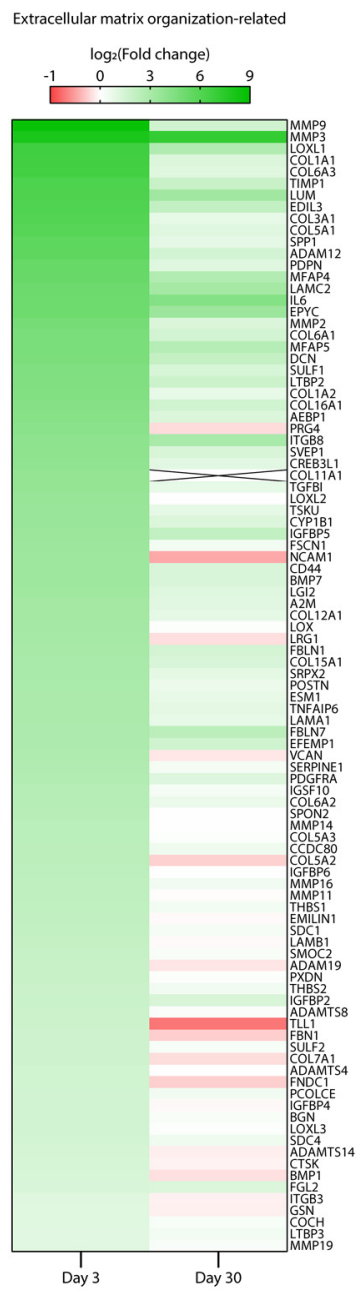

Figure 12. Transient activation of transcriptional programs related to vascular remodeling in peri-infarct endothelial cells in a mouse stroke model. Volcano plot showing upregulated (green) and downregulated (red) genes in peri-infarct endothelial cells $3 \mathrm{~d}(\boldsymbol{A})$ and $30 \mathrm{~d}(\boldsymbol{B})$ after middle cerebral artery occlusion relative to sham animals. $\boldsymbol{C}$, Heatmap comparison of enriched gene ontology terms among upregulated genes between 3 and $30 \mathrm{~d}$ after ischemia. Clustering is based on overlap of gene sets. Heatmap comparisons of expression of angiogenesis-related $(\boldsymbol{D})$ and extracellular matrix organization-related genes $(\boldsymbol{E})$ at 3 and $30 \mathrm{~d}$ after ischemia relative to sham. Genes are sorted by fold change relative to sham at $3 \mathrm{~d}$. Fold change was not calculated for one gene (COL11A1, black bars) at $30 \mathrm{~d}$ after ischemia because of very low read counts.

flow likely facilitates behavioral recovery through two mechanisms. First, the resolution of metabolic stress likely enables proper neuronal function in tissue with otherwise depressed activity (Carmichael et al., 2004; Summers et al., 2017; He et al., 
2020). Second, adequate blood flow is required for neural repair, including behaviorally relevant synaptic remodeling (Mostany et al., 2010; Kim et al., 2018; Clark et al., 2019a). Thus, we suggest that the restoration of blood flow via vascular plasticity contributes to resolution of abnormalities in neuronal function, promotes neural repair, and facilitates behavioral recovery.

The extent of vascular plasticity in peri-infarct regions likely depends on several factors. Occlusion of a penetrating arteriole causes progressive pathology over the course of hours in downstream capillaries, including constriction and reduced flow (Taylor et al., 2016). The extent to which microvascular flow is reduced, initial and residual flow in the occluded arteriole, and the ability of nearby penetrating arterioles and microvasculature to compensate for reduced blood flow all contribute to the severity of ischemia and infarct growth (Schaffer et al., 2006; Blinder et al., 2010; Taylor et al., 2016). Our finding that the initial severity of ischemia was associated with ensuing capillary formation suggests that capillary formation is most prevalent in regions where the residual vascular network poorly compensates for reduced flow. By contrast, regions in which blood flow can be maintained and restored by residual vessels likely undergo little vascular plasticity. Thus, we would expect the prevalence of vascular plasticity and its contributions to recovery to vary with the severity of peri-infarct blood flow deficits and the size of the ischemic penumbra.

Acute increases in the incidence of capillary stalls were recently reported in the ischemic penumbra within $24 \mathrm{~h}$ after distal middle cerebral artery occlusion (Erdener et al., 2020). We unexpectedly found an $\sim 10$-fold increase in their incidence in peri-infarct cortex at 4 weeks after infarct relative to the incidence in cortex of uninjured mice. Although we found that parenchymal blood flow averaged over relatively large volumes generally returned to normal in peri-infarct cortex by day 7 , stroke causes persistent neuronal dysfunction and hypometabolism in peri-infarct regions (Carmichael et al., 2004; Brown et al., 2009; Summers et al., 2017). In addition, long-lasting perturbations in neurovascular coupling after cortical infarcts were recently reported after even very small infarcts (He et al., 2020). We suggest that chronic impairments in microvascular blood flow, including capillary stalls, could contribute to these features of poststroke pathophysiology. Interestingly, anti-Ly6G antibodies, targeting neutrophils, have been demonstrated to at least transiently reduce the incidence of capillary stalls after acute experimental stroke and in an Alzheimer's disease model (Cruz Hernández et al., 2019; Erdener et al., 2020). Such a therapy could reduce chronic capillary stalls after stroke if neutrophil plugs are the cause. Alternatively, or perhaps in addition, injury could induce chronic contraction of capillary pericytes that increases the likelihood of stalls by reducing capillary diameter (Yemisci et al., 2009; Li et al., 2017; Hartmann et al., 2020). An in-depth study of the progression and cause of peri-infarct capillary stalls is warranted.

The stability of vascular structure in the uninjured brain and the short temporal window of plasticity after stroke suggest that vascular structure is resistant to change. We found that gene expression changes in peri-infarct endothelial cells were transient, coinciding with the window of structural remodeling. Two previous studies similarly showed that angiogenesis-related genes are upregulated in bulk peri-infarct tissue over a similar time course (Buga et al., 2014; Rust et al., 2019a), corroborating the transient nature of vascular remodeling-related gene expression changes. These findings suggest a natural limitation in spontaneous vascular repair. Subsequently, therapeutic enhancement of vascular plasticity is a potential treatment strategy. Indeed, several treatments shown to improve behavioral recovery in rodent models have also been associated with increased periinfarct vascular density (Ergul et al., 2012; López-Valdés et al., 2014; Nih et al., 2018; Rust et al., 2019a).

Therapeutic enhancement of vascular growth must be carefully balanced with excessive blood-brain barrier leakage (Weis and Cheresh, 2005). For example, exogenous VEGF promotes angiogenesis but also disrupts barrier function (Weis and Cheresh, 2005; Ergul et al., 2012). Vascular guidance cues are another potential therapeutic target that might avoid unintended effects on vascular permeability (Wälchli et al., 2013; Rust et al., 2019c). Recently, it was demonstrated that anti-Nogo-A antibody treatment, which is known to augment neuronal plasticity (Lee et al., 2004), increased vascular density and improved behavioral outcome in a mouse stroke model without exacerbating vascular permeability (Rust et al., 2019a,b). However, because of significant overlap of the array of guidance molecules that act on neuronal growth cones and endothelial tip cells (Lee et al., 2004; Carmeliet and Tessier-Lavigne, 2005; Wälchli et al., 2013; Rust et al., 2019b), it is difficult to disentangle whether beneficial effects on recovery of guidance molecule manipulations can be attributed to neuronal or vascular plasticity, or both.

Given that the majority of strokes occur in an aged population (Feigin et al., 2014), understanding how age influences the vascular response to stroke has significant translational relevance. Harb et al. (2013) demonstrated an age-related diminishment in the angiogenic response to long-term hypoxia, which suggests that the aged brain is particularly resistant to vascular plasticity. A comparison of gene expression in bulk peri-infarct tissue between 3-4- and 19-20-month-old rats revealed a marked age-related perturbation in the expression of many angiogenesis-related genes (Buga et al., 2014). Specifically, there was a delayed and often diminished upregulation of genes related to vascular sprouting and extracellular matrix remodeling in aged rats. These findings suggest that the window of vascular plasticity may be delayed and diminished in older animals relative to the young adults we studied. Histologic measurements have found relatively reduced peri-infarct vessel density in older rodents and humans (Szpak et al., 1999; Ergul et al., 2012; Buga et al., 2014). Based on our current findings, we speculate that the blunting of poststroke vascular plasticity with age could contribute to worse functional outcome.

We demonstrated that the vascular remodeling response following stroke involves a window of microvascular structural plasticity. Our data support that vascular structural plasticity is a mechanism that contributes to the restoration of blood flow in residual peri-infarct cortex. We hypothesize that reestablished blood flow permits neural repair and normal neuronal function, which facilitate behavioral recovery. Chronic localized perturbations in microvascular flow because of capillary stalls may hinder recovery by limiting repair and disrupting neuronal function.

\section{References}

Blinder P, Shih AY, Rafie C, Kleinfeld D (2010) Topological basis for the robust distribution of blood to rodent neocortex. Proc Natl Acad Sci USA 107:12670-12675.

Brown CE, Aminoltejari K, Erb H, Winship IR, Murphy TH (2009) In vivo voltage-sensitive dye imaging in adult mice reveals that somatosensory maps lost to stroke are replaced over weeks by new structural and functional circuits with prolonged modes of activation within both the periinfarct zone and distant sites. J Neurosci 29:1719-1734. 
Brown CE, Li P, Boyd JD, Delaney KR, Murphy TH (2007) Extensive turnover of dendritic spines and vascular remodeling in cortical tissues recovering from stroke. J Neurosci 27:4101-4109.

Buga AM, Margaritescu C, Scholz CJ, Radu E, Zelenak C, Popa-Wagner A (2014) Transcriptomics of post-stroke angiogenesis in the aged brain. Front Aging Neurosci 6:44-20.

Carmeliet P, Tessier-Lavigne M (2005) Common mechanisms of nerve and blood vessel wiring. Nature 436:193-200.

Carmichael ST (2006) Cellular and molecular mechanisms of neural repair after stroke: making waves. Ann Neurol 59:735-742.

Carmichael ST, Tatsukawa K, Katsman D, Tsuyuguchi N, Kornblum HI (2004) Evolution of diaschisis in a focal stroke model. Stroke 35:758-763.

Clark TA, Sullender C, Jacob D, Zuo Y, Dunn AK, Jones TA (2019a) Rehabilitative training interacts with ischemia-instigated spine dynamics to promote a lasting population of new synapses in peri-infarct motor cortex. J Neurosci 39:8471-8483.

Clark TA, Sullender C, Kazmi SM, Speetles BL, Williamson MR, Palmberg DM, Dunn AK, Jones TA (2019b) Artery targeted photothrombosis widens the vascular penumbra, instigates peri-infarct neovascularization and models forelimb impairments. Sci Rep 9:2323.

Cruz Hernández JC, Bracko O, Kersbergen CJ, Muse V, Haft-Javaherian M, Berg M, Park L, Vinarcsik LK, Ivasyk I, Rivera DA, Kang Y, CortesCanteli M, Peyrounette M, Doyeux V, Smith A, Zhou J, Otte G, Beverly JD, Davenport E, Davit Y, et al. (2019) Neutrophil adhesion in brain capillaries reduces cortical blood flow and impairs memory function in Alzheimer's disease mouse models. Nat Neurosci 22:413-420.

Dijkhuizen RM, Singhal AB, Mandeville JB, Wu O, Halpern EF, Finklestein SP, Rosen BR, Lo EH (2003) Correlation between brain reorganization, ischemic damage, and neurologic status after transient focal cerebral ischemia in rats: a functional magnetic resonance imaging study. J Neurosci 23:510-517.

Erdener ŞE, Tang J, Sajjadi A, Kılıç K, Kura S, Schaffer CB, Boas DA (2019) Spatio-temporal dynamics of cerebral capillary segments with stalling red blood cells. J Cereb Blood Flow Metab 39:886-900.

Erdener ŞE, Tang J, Kılıç K, Postnov D, Giblin JT, Kura S, Chen IC, Vayisoğlu T, Sakadzõić S, Schaffer CB, Boas DA (2020) Dynamic capillary stalls in reperfused ischemic penumbra contribute to injury: a hyperacute role for neutrophils in persistent traffic jams. J Cereb Blood Flow Metab. Advance online publication. Retrieved April 1, 2020. DOI: 10.1177/0271678X20914179. .

Ergul A, Alhusban A, Fagan SC (2012) Angiogenesis: a harmonized target for recovery after stroke. Stroke 43:2270-2274.

Feigin VL, Forouzanfar MH, Krishnamurthi R, Mensah GA, Connor M, Bennett DA, Moran AE, Sacco RL, Anderson L, Truelsen T, O'Donnell M, Venketasubramanian N, Barker-Collo S, Lawes CM, Wang W, Shinohara Y, Witt E, Ezzati M, Naghavi M, Murray C (2014) Global and regional burden of stroke during 1990-2010: findings from the Global Burden of Disease Study 2010. Lancet 383:245-255.

Harb R, Whiteus C, Freitas C, Grutzendler J (2013) In vivo imaging of cerebral microvascular plasticity from birth to death. J Cereb Blood Flow Metab 33:146-156.

Hartmann DA, Berthiaume AA, Grant RI, Harill SA, Noonan T, Costello J, Tieu T, McDowell K, Faino A, Kelly A, Shih AY (2020) Brain capillary pericytes exert a substantial but slow influence on blood flow. BioRxiv 008763. DOI: 10.1101/2020.03.26.008763.

He F, Sullender C, Zhu H, Williamson MR, Li X, Zhao Z, Jones TA, Xie C, Dunn AK, Luan L (2020) Multimodal mapping of neural activity and cerebral blood flow reveals long-lasting neurovascular dissociations after small-scale strokes. Sci Adv 6:eaba1933.

Hermer-Vazquez L, Hermer-Vazquez R, Chapin JK (2007) The reach-tograsp-food task for rats: a rare case of modularity in animal behavior? Behav Brain Res 177:322-328.

Holtmaat A, Bonhoeffer T, Chow DK, Chuckowree J, Paola V, De Keck T, Knott G, Lee WA, Mostany R, Hofer SB, Hu M, Mrsic-Flogel TD, Nedivi E, Portera-Cailliau C, Svoboda K, Trachtenberg JT, Wilbrecht L (2009) Long-term, high-resolution imaging in the mouse neocortex through a chronic cranial window. Nat Protoc 4:1128-1144.

Jones TA, Adkins DL (2015) Motor system reorganization after stroke: stimulating and training toward perfection. Physiology (Bethesda) 30:358370 .
Kazmi SM, Parthasarthy AB, Song NE, Jones TA, Dunn AK (2013) Chronic imaging of cortical blood flow using Multi-Exposure Speckle Imaging. J Cereb Blood Flow Metab 33:798-808.

Kim SY, Hsu JE, Husbands LC, Kleim JA, Jones TA (2018) Coordinated plasticity of synapses and astrocytes underlies practice-driven functional vicariation in peri-infarct motor cortex. J Neurosci 38:93-107.

Kirst C, Skriabine S, Vieites-Prado A, Topilko T, Bertin P, Gerschenfeld G, Verny F, Topilko P, Michalski N, Tessier-Lavigne M, Renier N (2020) Mapping the fine-scale organization and plasticity of the brain vasculature. Cell 180:780-795.e25.

Klein A, Sacrey LA, Whishaw IQ, Dunnett SB (2012) The use of rodent skilled reaching as a translational model for investigating brain damage and disease. Neurosci Biobehav Rev 36:1030-1042.

Korn C, Augustin HG (2015) Mechanisms of vessel pruning and regression. Dev Cell 34:5-17.

Krupinski J, Kaluza J, Kumar P, Kumar S, Wang JM (1994) Role of angiogenesis in patients with cerebral ischemic stroke. Stroke 25:1794-1798.

Lake EM, Bazzigaluppi P, Mester J, Thomason LA, Janik R, Brown M, McLaurin JA, Carlen PL, Corbett D, Stanisz GJ, Stefanovic B (2017) Neurovascular unit remodelling in the subacute stage of stroke recovery. Neuroimage 146:869-882.

Lawrence ES, Coshall C, Dundas R, Stewart J, Rudd AG, Howard R, Wolfe CDA (2001) Estimates of the prevalence of acute stroke impairments and disability in a multiethnic population. Stroke 32:1279-1284.

Lee JK, Kim JE, Sivula M, Strittmatter SM (2004) Nogo receptor antagonism promotes stroke recovery by enhancing axonal plasticity. J Neurosci 24:6209-6217.

Li Y, Lucas-Osma AM, Black S, Bandet MV, Stephens MJ, Vavrek R, Sanelli L, Fenrich KK, Di Narzo AF, Dracheva S, Winship IR, Fouad K, Bennett DJ (2017) Pericytes impair capillary blood flow and motor function after chronic spinal cord injury. Nat Med 23:733-741.

Liman TG, Endres M (2012) New vessels after stroke: postischemic neovascularization and regeneration. Cerebrovasc Dis 33:492-499.

Lin TN, Sun SW, Cheung WM, Li F, Chang C (2002) Dynamic changes in cerebral blood flow and angiogenesis after transient focal cerebral ischemia in rats: evaluation with serial magnetic resonance imaging. Stroke 33:2985-2991.

Liu J, Wang Y, Akamatsu Y, Lee CC, Stetler RA, Lawton MT, Yang GY (2014) Vascular remodeling after ischemic stroke: mechanisms and therapeutic potentials. Prog Neurobiol 115:138-156.

López-Valdés HE, Clarkson AN, Ao Y, Charles AC, Carmichael ST, Sofroniew MV, Brennan KC (2014) Memantine enhances recovery from stroke. Stroke 45:2093-2100.

Martín A, Macé E, Boisgard R, Montaldo G, Thézé B, Tanter M, Tavitian B (2012) Imaging of perfusion, angiogenesis, and tissue elasticity after stroke. J Cereb Blood Flow Metab 32:1496-1507.

Mostany R, Chowdhury TG, Johnston DG, Portonovo SA, Carmichael ST, Portera-Cailliau C (2010) Local hemodynamics dictate longterm dendritic plasticity in peri-infarct cortex. J Neurosci 30: 14116-14126.

Munji RN, Soung AL, Weiner GA, Sohet F, Semple BD, Trivedi A, Gimlin K, Kotoda M, Korai M, Aydin S, Batugal A, Cabangcala AC, Schupp PG, Oldham MC, Hashimoto T, Noble-Haeusslein LJ, Daneman R (2019) Profiling the mouse brain endothelial transcriptome in health and disease models reveals a core blood-brain barrier dysfunction module. Nat Neurosci 22:1892-1902.

Nih LR, Gojgini S, Carmichael ST, Segura T (2018) Dual-function injectable angiogenic biomaterial for the repair of brain tissue following stroke. Nat Mater 17:642-651.

Reeson P, Choi K, Brown CE (2018) VEGF signaling regulates the fate of obstructed capillaries in mouse cortex. eLife 7:1-26.

Robertson RT, Levine ST, Haynes SM, Gutierrez P, Baratta JL, Tan Z, Longmuir KJ (2015) Use of labeled tomato lectin for imaging vasculature structures. Histochem Cell Biol 143:225-234.

Rust R, Grönnert L, Gantner C, Enzler A, Mulders G, Weber RZ (2019a) Nogo-A targeted therapy promotes vascular repair and functional recovery following stroke. Proc Natl Acad Sci USA 116:14270-14279.

Rust R, Grönnert L, Weber RZ, Mulders G, Schwab ME (2019b) Refueling the ischemic CNS: guidance molecules for vascular repair. Trends Neurosci 42:644-656.

Rust R, Weber RZ, Grönnert L, Mulders G, Maurer MA, Hofer AS, Sartori AM, Schwab ME (2019c) Anti-Nogo-A antibodies prevent 
vascular leakage and act as pro-angiogenic factors following stroke. Sci Rep 9:10.

Schaffer CB, Friedman B, Nishimura N, Schroeder LF, Tsai PS, Ebner FF, Lyden PD, Kleinfeld D (2006) Two-photon imaging of cortical surface microvessels reveals a robust redistribution in blood flow after vascular occlusion. PLoS Biol 4:e22.

Schager B, Brown CE (2020) Susceptibility to capillary plugging can predict brain region specific vessel loss with aging. J Cereb Blood Flow Metab Advance online publication. Retrieved January 5, 2020. DOI: 10.1177/ $0271678 X 19895245$

Schrandt CJ, Kazmi SM, Jones TA, Dunn AK (2015) Chronic monitoring of vascular progression after ischemic stroke using multiexposure speckle imaging and two-photon fluorescence microscopy. J Cereb Blood Flow Metab 35:933-942.

Shih AY, Blinder P, Tsai PS, Friedman B, Stanley G, Lyden PD, Kleinfeld D (2013) The smallest stroke: occlusion of one penetrating vessel leads to infarction and a cognitive deficit. Nat Neurosci 16:55-63.

Summers PM, Hartmann DA, Hui ES, Nie X, Deardorff RL, McKinnon ET, Helpern JA, Jensen JH, Shih AY (2017) Functional deficits induced by cortical microinfarcts. J Cereb Blood Flow Metab 37:3599-3614.

Szpak GM, Lechowicz W, Lewandowska E, Bertrand E, Wierzba-Bobrowicz T, Dymecki J (1999) Border zone neovascularization in cerebral ischemic infarct. Folia Neuropathol 37:264-268.

Taylor ZJ, Hui ES, Watson AN, Nie X, Deardorff RL, Jensen JH, Helpern JA, Shih AY (2016) Microvascular basis for growth of small infarcts following occlusion of single penetrating arterioles in mouse cortex. J Cereb Blood Flow Metab 36:1357-1373.

Tennant KA, Adkins DL, Donlan NA, Asay AL, Thomas N, Kleim JA, Jones TA (2011) The organization of the forelimb representation of the C57BL/
6 mouse motor cortex as defined by intracortical microstimulation and cytoarchitecture. Cereb Cortex 21:865-876.

Tennant KA, Taylor SL, White ER, Brown CE (2017) Optogenetic rewiring of thalamocortical circuits to restore function in the stroke injured brain. Nat Commun 8:15879-15814.

Villringer A, Them A, Lindauer U, Einhäupl K, Dirnagl U (1994) Capillary perfusion of the rat brain cortex: an in vivo confocal microscopy study. Circ Res 75:55-62.

Wälchli T, Mateos JM, Weinman O, Babic D, Regli L, Hoerstrup SP, Gerhardt H, Schwab ME, Vogel J (2015) Quantitative assessment of angiogenesis, perfused blood vessels and endothelial tip cells in the postnatal mouse brain. Nat Protoc 10:53-74.

Wälchli T, Pernet V, Weinmann O, Shiu JY, Guzik-Kornacka A, Decrey G, Yüksel D, Schneider H, Vogel J, Ingber DE, Vogel V, Frei K, Schwab ME (2013) Nogo-A is a negative regulator of CNS angiogenesis. Proc Natl Acad Sci USA 110:E1943-E1952.

Weis SM, Cheresh DA (2005) Pathophysiological consequences of VEGFinduced vascular permeability. Nature 437:497-504.

Xu T, Yu X, Perlik AJ, Tobin WF, Zweig JA, Tennant K, Jones T, Zuo Y (2009) Rapid formation and selective stabilization of synapses for enduring motor memories. Nature 462:915-919.

Yanev P, Dijkhuizen RM (2012) In vivo imaging of neurovascular remodeling after stroke. Stroke 43:3436-3441.

Yemisci M, Gursoy-Ozdemir Y, Vural A, Can A, Topalkara K, Dalkara T (2009) Pericyte contraction induced by oxidative-nitrative stress impairs capillary reflow despite successful opening of an occluded cerebral artery. Nat Med 15:1031-1037.

Zhou Y, Zhou B, Pache L, Chang M, Khodabakhshi AH, Tanaseichuk O, Benner C, Chanda SK (2019) Metascape provides a biologist-oriented resource for the analysis of systems-level datasets. Nat Commun 10:1523. 